\title{
Joint Radar-Communication Relying on NLFM-MSK Design
}

\author{
Hao Ma $\mathbb{D}$, Jun Wang $\mathbb{D}$, Xin Sun, TianWei Hou $\mathbb{D}$, and WenXin Jin $\mathbb{D}$ \\ School of Electronic and Information Engineering, Beijing Jiaotong University, Beijing, China \\ Correspondence should be addressed to Jun Wang; wangjun1@bjtu.edu.cn
}

Received 6 July 2021; Revised 16 December 2021; Accepted 30 December 2021; Published 22 January 2022

Academic Editor: Luis Castedo

\begin{abstract}
Copyright (C) 2022 Hao Ma et al. This is an open access article distributed under the Creative Commons Attribution License, which permits unrestricted use, distribution, and reproduction in any medium, provided the original work is properly cited.
\end{abstract}

\begin{abstract}
The existing joint radar-communication (JRC) based on linear frequency modulation (LFM) is imperfect because of high autocorrelation sidelobe and poor nearby target detection performance. This paper proposes a JRC signal based on the nonLFM (NLFM) wave by using minimum shift keying (MSK). Then, this paper analyzes the performance of ambiguity function (AF) characteristics, detection probability, time-frequency characteristics, and communication rate for the proposed JRC signal. It is concluded that the proposed JRC signal has both excellent radar and communication characteristics. Under the condition of low data volume, the simulation results illustrate that (i) compared with the existing LFM-MSK signal, our JRC signal's sidelobe level of the time AF is significantly lower; the range resolution is improved significantly; (ii) the proposed JRC signal has no loss on velocity resolution and detection probability; and (iii) the proposed JRC signal has excellent time-frequency characteristics.
\end{abstract}

\section{Introduction}

Wireless communication and radar detection, which are widely used in modern society, have different application scenarios, architectures, and frequency bands. The growing number of wireless terminals and radar equipment brings a series of problems such as spectrum overlap, functional interference, and energy loss [1-3]. In this situation, limited wireless spectrum resources have become more and more valuable, making the reuse of spectrum resources extremely urgent. Based on this application background, the joint radar-communication (JRC) technology is gaining more and more attention [4-6]. As shown in Figure 1, the JRC system has greatly alleviated the problems of spectrum shortage, multiplatform interference, antenna reuse, and excessive energy consumption. The application of JRC has also expanded from the military field to the civil fields, and the waveform design method is the key part of the JRC technology.

In recent years, several methods have been proposed to complete the advanced JRC waveform design, and researchers have achieved a lot both theoretically and practically. Through the analysis of the existing literature, it can be con- cluded that approaches of JRC system design can be mainly divided into three types: JRC technology based on time division, JRC technology based on multibeam sharing, and JRC technology based on integrated wave.

JRC time division is a simple way of integration technologies, which means that the radar and communication systems use the same hardware platform at different time slots [7]. In this method, the original waveforms of radar and communication do not need to be changed, and different waveforms are emitted in different time slots. For JRC system, only the reasonable division of time gap needs to be considered; the two functions alternate in the time domain and there is no mutual interference [8]. JRC system can also realize the multiplexing of antenna and transceiver equipment through the gate switch. JRC time division is shown in Figure 2. After the radar detects the target, the communication information is transmitted. After the communication, the radar beam detects again, and the detection time and communication time can be adjusted flexibly. Although the existing platform resources can be fully utilized, the low efficiency is still a problem. The radar and communication cannot be operated at the same time, which causes the detection blind area. 


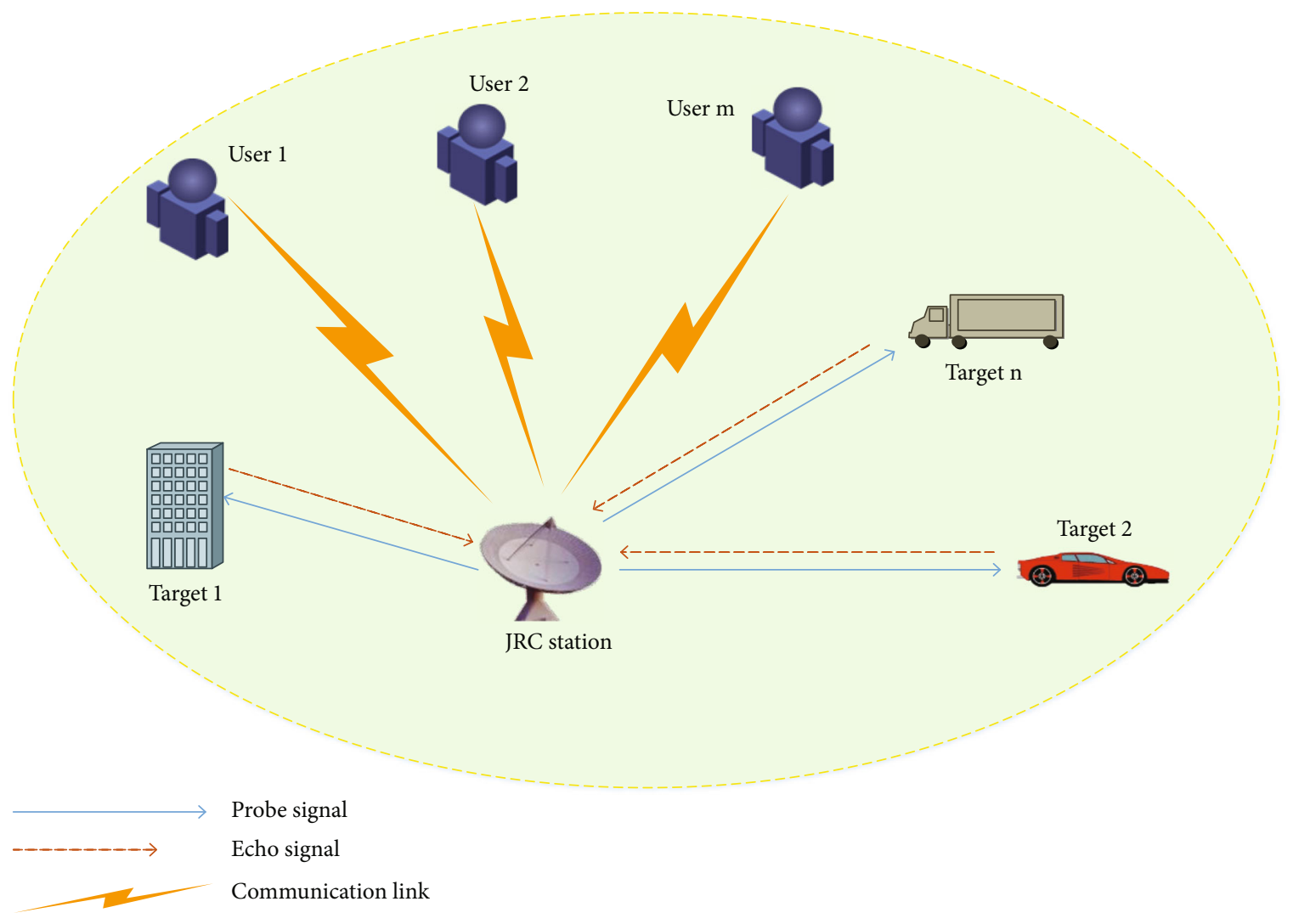

Figure 1: The diagram of JRC system.

JRC time-division technology

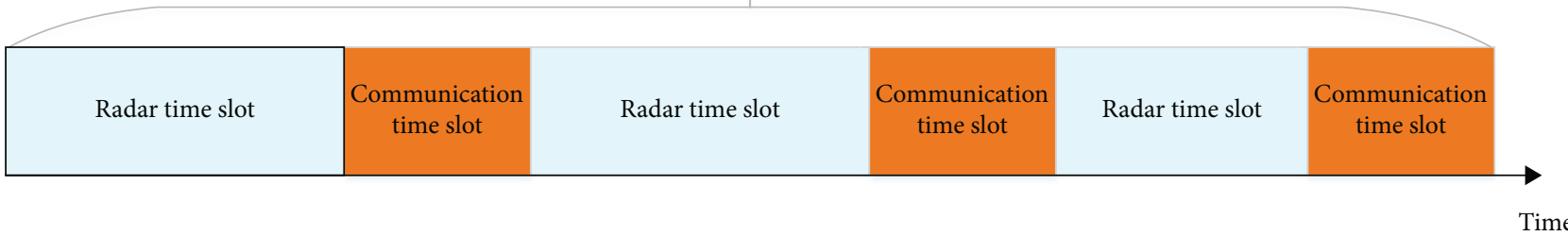

Figure 2: The diagram of JRC time division.

In JRC multibeam sharing technology, JRC systems generate multibeam signals by designing the layout, antenna number, and direction of multiple input multiple output (MIMO) array antennas. As shown in Figure 3(a), JRC system uses different beam signals to realize the radar and communication, respectively [9]. JRC multibeam sharing technology is flexible and has good degree of freedom, but it is prone to functional interference. So the main design objective is to synthesize radar performance and communication performance and to optimize the design of the synthesized performance by using optimization theory [10]. Another JRC multibeam sharing technology uses radar main lobe to achieve detection performance and uses radar waveform sidelobe to communicate [11], which is shown in Figure 3(b). This method can effectively reduce the interference between radar main lobe and communication sidelobe, but there are many waveform limitations.
Different from the first two technologies, the integrated wave technology completely realizes the nonseparation transmission of radar signal and communication signal, and there is no obvious separation of the two signals in the space, time, and frequency domains. In this paper, we pay close attention on JRC system of integrated wave. Linear frequency modulation (LFM) wave is a popular waveform in the fields of radar, detection, and sonar, which has excellent range resolution and velocity resolution, and many JRC technologies adopt LFM wave as the seminal waveform. Literature [12-14] had promoted LFM-MSK signal as JRC waveform and analyzed the ambiguity function (AF) characteristics; researchers also proposed methods to reduce the energy leakage and the increase communication throughout JRC waveform. In literature $[15,16]$, spread spectrum technology was introduced into the JRC waveform design, and combined with small angle BPSK and LFM waves, the 


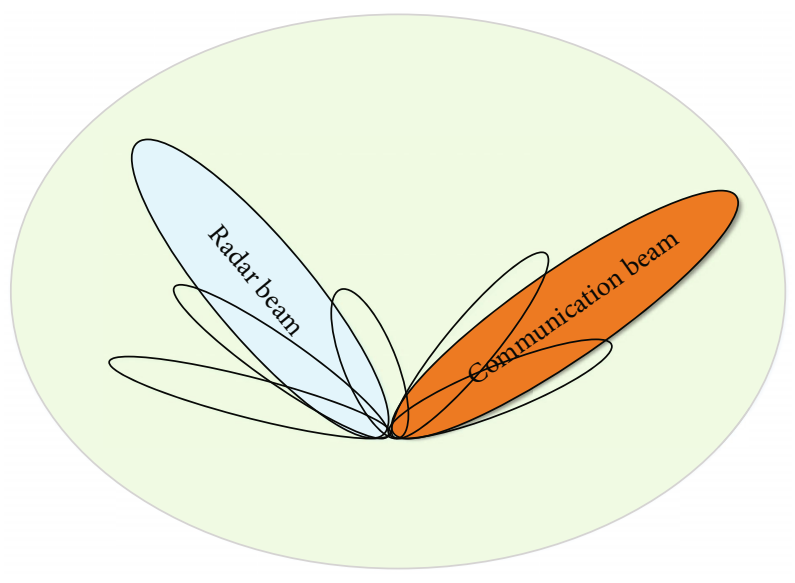

(a)

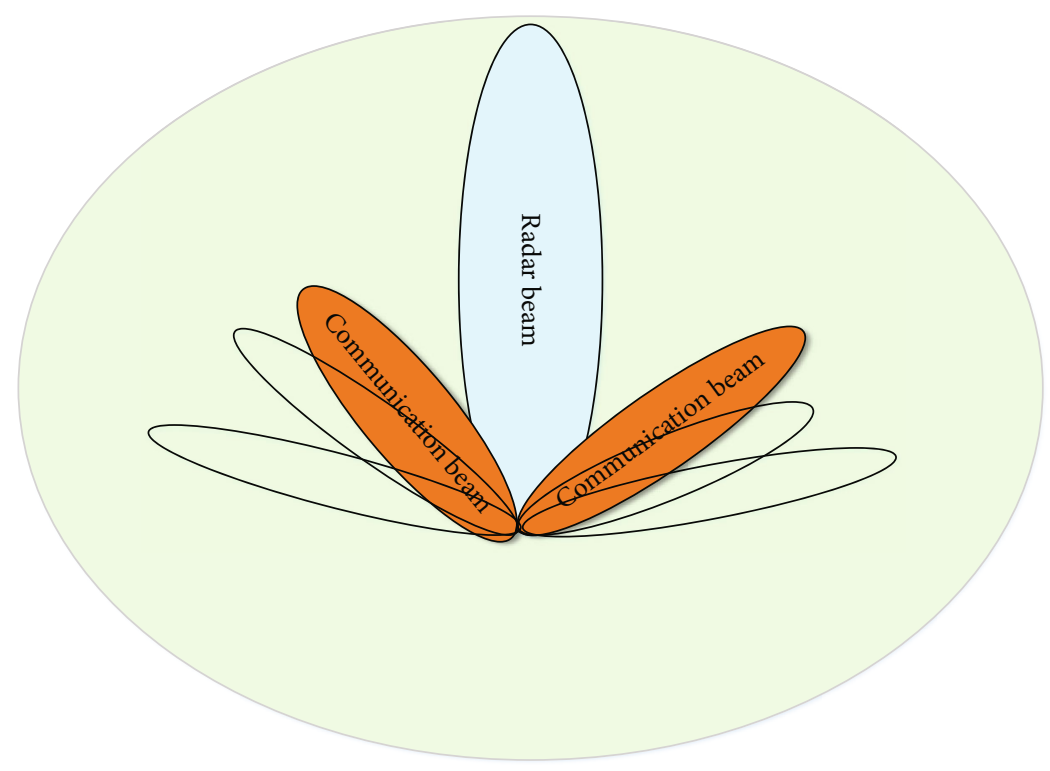

(b)

FIGURE 3: The diagram of JRC multibeam sharing: (a) different beams for radar and communication, respectively; (b) main lobe for radar and sidelobe for communication.

integrated signal could achieve a higher communication rate while ensuring the detection performance of the radar. Orthogonal frequency division multiplexing (OFDM) technology has unique advantages in communication signal design and signal processing. Although its inherent features affect the nonlinear power amplification of radar platform, OFDM is also widely introduced into the field of JRC waveform. Literature [17-19] had proposed JRC signal based on OFDM technology and LFM signal and analyzed the JRC signal from the perspectives of AF, peak-to-average power ratio, detection accuracy, etc. A large number of theoretical discussions and improvement studies had been carried out. MIMO technology, widely used in 5G communication system, has many advantages such as diversity gain and large communication capacity. Therefore, the JRC based on MIMO technology has also received extensive attention from academia and industry. Under the optimizations' constraints, literature [20-23] proposed the beamforming technology to realize MIMO communication and radar detection, and some algorithms were designed to solve the nonconvex problems in the JRC waveform. The LFM-CPM signal, at the basis of LFM-MSK, greatly promoted the application of JRC technology in the field of intelligent transportation $[24,25]$. The early JRC technologies had achieved some breakthroughs and developments in the fields of radar and communication. However, in the seminal LFM base, due to the embedded communication bits, the LFM-MSK signal inevitably has the problem of excessively high sidelobe of auto correlation function, which mask the nearby targets in radar detection.

In order to solve the nearby multitarget detecting problem, a novel JRC waveform based on non-LFM (NLFM) radar signal is proposed in this paper, called NLFM-MSK. Our JRC signal has the advantages of constant envelope, continuous phase, and low sidelobe of time AF, which effectively improves the accuracy of nearby multitarget measurement. Through theoretical analysis and computer simulation, it is proved that our JRC signal has better radar performance than the LFM- 
MSK signal mentioned in literature [11] under the low data volume condition. Our contributions are summarized as follows:

(1) We propose a novel JRC signal model of NLFMMSK waveform and analyze its time-frequency characteristics, autocorrelation characteristics, and timedomain signal characteristics

(2) We analyze the radar performance criteria and communication performance criteria, and we derive the mathematical equations of the relevant criteria

(3) The mathematical derivation and simplified analysis of the AF of our JRC signal are carried out, and the superiority of the proposed signal is proved theoretically in the low communication data condition

(4) The AF characteristics, detection probability, timefrequency characteristics, and communication rate of our JRC signal are analyzed and simulated, and the results of performance analysis are verified

The rest of the paper is organized as follows. Section 2 presents our JRC model and derives mathematical expressions. In Section 3, we derive the AF equations of our JRC, detection probability and time-frequency characteristics, and we analyze the communication and radar performances. Section 4 provides computer simulation, and Section 3verifies our discussion. Finally, the conclusion is drawn in Section 5.

Notation: in the paper, the NLFM waveform is denoted by $s_{\mathrm{NLFM}}(t)$, the baseband continuous phase modulation (CPM) waveform is denoted by $s_{\mathrm{CPM}}(t)$, the baseband minimum shift keying (MSK) waveform is denoted by $s_{\mathrm{MSK}}(t)$, and the JRC waveform is denoted by $s(t) . B$ is the signal bandwidth, $T_{p}$ is the time width of signal, and $T_{s}$ is the time width of bit. We assume $B=10 \mathrm{MHz}$ and $T_{p}=40 \mu$ s.

\section{System Model}

LFM signal is suitable for most radar signal amplification systems, and its frequency changes linearly with time. However, due to the inherent characteristics, the autocorrelation sidelobe of LFM wave is high up to $-13.26 \mathrm{~dB}$ [26], which affects the near multitarget detection. In practical application, a windowing function can be used to suppress the high autocorrelation sidelobe, but the method introduces the main lobe broadening extremely, SNR decreasing and other problems. The early JRC waveforms composed of LFM $[12-19,24,25]$ inherit the disadvantages of LFM. Furthermore, due to the communication data, the sidelobe of time AF for such a JRC signal becomes high and the range resolution performance deteriorates. In order to solve this problem and improve the range resolution of the early LFM integrated signal, NLFM signal is proposed as the seminal signal to generate novel JRC signal. The NLFM signal has the advantage of offering a low autocorrelation sidelobe. On the basis of the above analysis, this paper takes NLFM signal as the carrier wave and MSK as the modulation wave to design an integrated NLFM-MSK signal. Under the low data volume condition, this JRC signal cannot only realize the target detection and wireless communication but also has perfect AF performance, low range sidelobe, and gainful spectral characteristics.

2.1. NLFM Signal. NLFM waveform is a kind of complex signal with many signal models. The generation of NLFM is more complex than generation of LFM signal, and there are no precise mathematical expressions. In previous literature, the NLFM waveform was expressed by an approximate expression, and its theoretical basis was the classical stationary phase principle [26, 27]. At present, there are two methods to generate NLFM signal. (1) For the existing LFM signal, the altering frequency modulation function is adopted to produce NLFM signals. (2) Using the existing window function, the inverse method of window function is adopted to generate NLFM signal. Considering the convenience of signal generation, this paper adopts the second method to generate NLFM signal. The common window functions include Hamming window, Hanning window, and Blackman window. The autocorrelations of the three window functions are shown in Figure 4 and Table 1.

Through comparison, this paper adopts the Hamming window, with the lowest sidelobe level of the autocorrelation function, to generate NLFM signal. The expression of Hamming window function in frequency domain is [28]

$$
W(f)=\left\{\begin{array}{l}
(1-\alpha)-\alpha \cdot \cos \left(\frac{2 \cdot \pi \cdot f}{B}\right),|f| \leq \frac{B}{2}, \\
0,|f| \geq \frac{B}{2}
\end{array}\right.
$$

where $B$ is the signal bandwidth and $\alpha$ is a configuration parameter that in the paper is fixed to $\alpha=0.46$. If the window function $W(f)$ is integrated, we can obtain the group delay function of the signal, which can be expressed as

$$
T(f)=K \cdot \int_{-\infty}^{f} W(x) d x, \quad-\frac{B}{2}<f<\frac{B}{2},
$$

where $K$ is a constant coefficient, which we assume unitary $(K=1)$. The inverse of the group delay function $T(f)$, which is the expression of the time-domain function, can be obtained:

$$
f(t)=T^{-1}(f), \quad 0 \leq t \leq T_{p}
$$

where $T_{p}$ is the time width of signal. By integrating equation (3), its phase function can be obtained as follows:

$$
\phi(t)=2 \pi \int_{0}^{t} f(x) d x, \quad 0 \leq t \leq T_{p} .
$$

The time-domain expression of the final NLFM signal is

$$
s_{\mathrm{NLFM}}(t)=A \cdot \operatorname{rect}\left(\frac{t}{T_{p}}\right) \cdot \exp \left[j \cdot\left(2 \cdot \pi \cdot f_{c} \cdot t+\phi(t)\right)\right]
$$



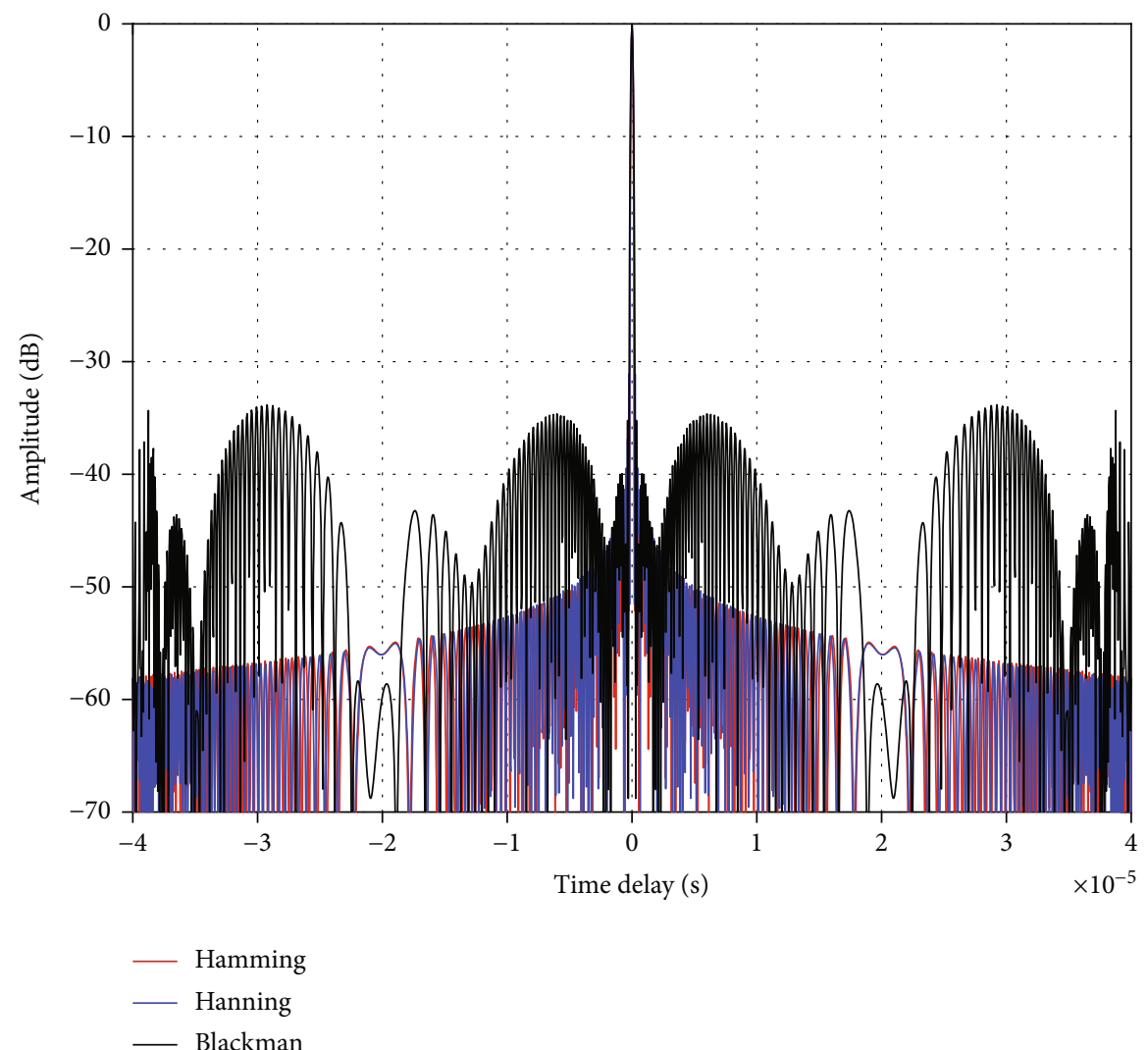

(a)
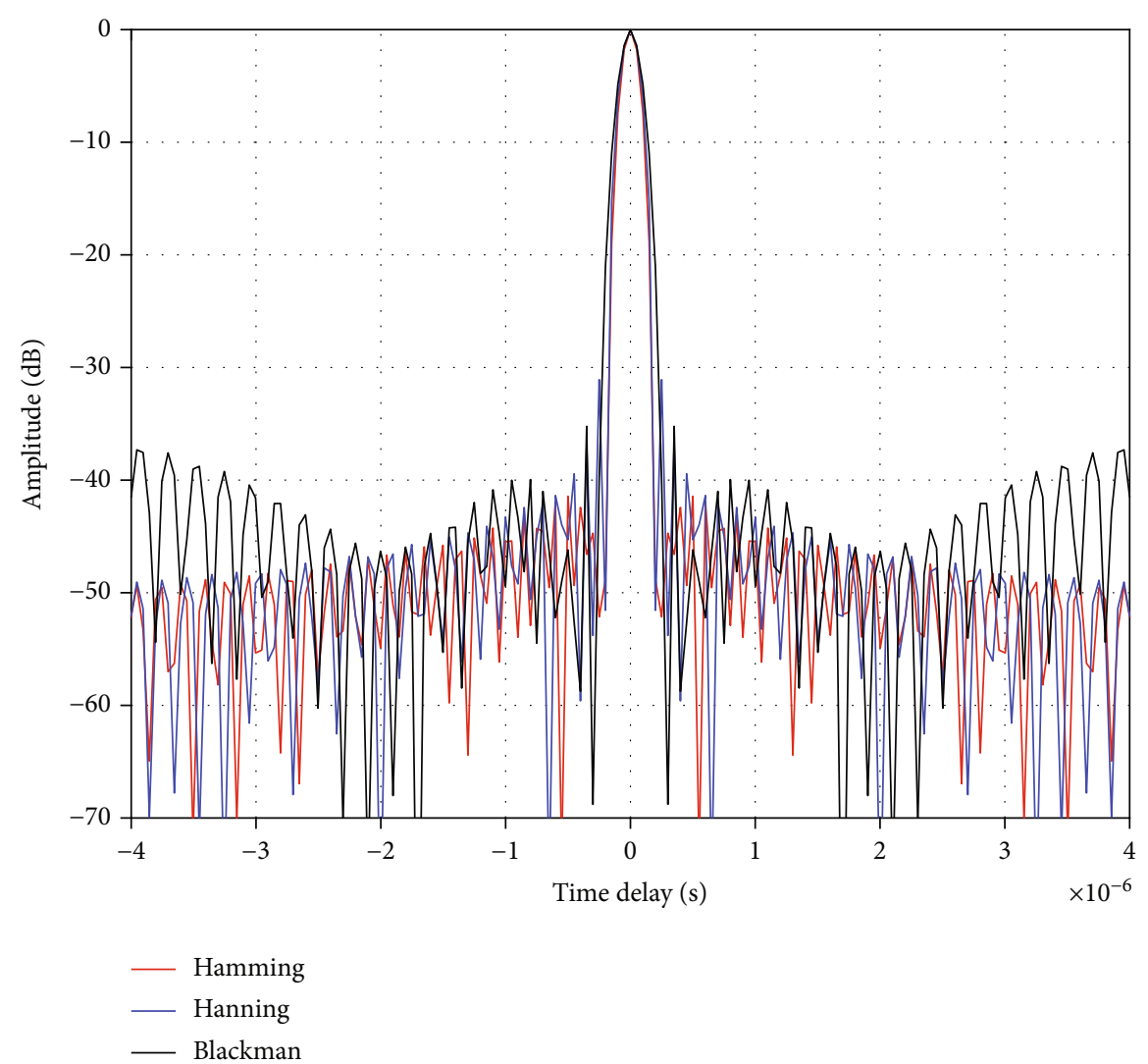

(b)

FIGURE 4: The autocorrelations for window functions: (a) global chart; (b) local chart. 
TABLE 1: Ratios of sidelobe peak to main lobe peak.

\begin{tabular}{lcccc}
\hline Signal & Hamming & Hanning & Blackman & LFM \\
\hline Ratio & $-42 \mathrm{~dB}$ & $-31 \mathrm{~dB}$ & $-35 \mathrm{~dB}$ & $-13.26 \mathrm{~dB}[25]$ \\
\hline
\end{tabular}

where $A$ is the amplitude of NLFM signal, $f_{c}$ is the initial frequency of NLFM signal, $\operatorname{rect}\left(t / T_{p}\right)$ is a rectangular window with duration $T_{p}$, and $\phi(t)$ is the phase without specific expression. From the design convenience consideration, we assume the amplitude $A=1$ and the initial frequency $f_{c}=0$ . So far, the time-domain expression of NLFM signal is completed according to the derivation of equations (1)-(5). The NLFM signal in the paper is

$$
s_{\mathrm{NLFM}}(t)=\operatorname{rect}\left(\frac{t}{T_{p}}\right) \cdot \exp [j \cdot \phi(t)]
$$

The comparison of autocorrelation characteristics between NLFM signals and LFM signal is shown in Figure 5. Under the same parameters, the autocorrelation function of NLFM signal has low sidelobes, and both the nearby target detection performance and the range detection performance are better than those of LFM signal.

2.2. MSK Modulation. The JRC technology usually introduces the communication data into the existing radar waveform, which inevitably leads to the spread of signal spectrum and the deterioration of the radar detection performance. The choice of the communication modulation is particularly important for the overall performance of the JRC signal. Considering several commonly used digital modulation modes, continuous phase modulation (CPM) mode, which is widely used in modern communication, has the advantages of constant signal envelope, continuous phase, and low bit error rate (BER). Hence, CPM modulation can be considered as the communication modulation of integrated signal design. The expression of baseband CPM is $[12,24]$

$$
s_{\mathrm{CPM}}(t)=A_{m} \cdot \operatorname{rect}\left(\frac{t}{T_{p}}\right) \cdot \exp (j \cdot(\theta(t, I)+\varphi))
$$

where $A_{m}$ is the signal amplitude and $\varphi$ is the initial phase of the code. $\theta(t, I)$ is the phase information after code modulation. Its expression is $[12,24]$

$$
\theta(t, I)=\sum_{n=0}^{N-1} \operatorname{rect}\left(\frac{t-n \cdot T_{s}}{T_{s}}\right) \cdot \theta\left(t, I_{n}\right)
$$

where $N$ is the number of codes carried by the MSK modulation signal and $T_{s}$ is the time width of bit, $T_{s}=T_{p} / N$. The phase of the $n$-th bit is expressed as [12, 24]

$$
\begin{aligned}
\theta\left(t, I_{n}\right)= & h \cdot \pi \cdot \sum_{k=-\infty}^{n-1} I_{k}+2 \cdot \pi \cdot h \cdot I_{n} \\
& \cdot q\left(t-n \cdot T_{s}\right), \quad n \cdot T_{s} \leq t<(n+1) \cdot T_{s},
\end{aligned}
$$

where $I_{n}$ represents the code sequence, $h$ is the modulation index, and $q(t)$ is the integral of the code impulse function $g(t)$. The functional relation satisfies $q(t)=\int_{0}^{t} g(\tau) d \tau$. The impulse function $g(t)$ is usually a rectangular function, a rising cosine function or a Gaussian pulse. In this paper, we assume that $g(t)$ is a rectangular impulse function.

Considering the convenience of the design, this paper finally decides to use MSK as the communication method of our JRC signal. MSK signal is a special form of binary CPM signal, with the characteristics of $h=0.5$ and $I_{n} \in\{ \pm 1\}$ [12-14].

The expression of baseband MSK is the same as (7)

$$
s_{\mathrm{MSK}}(t)=A_{m} \cdot \operatorname{rect}\left(\frac{t}{T_{p}}\right) \cdot \exp (j \cdot(\theta(t, I)+\varphi)) .
$$

The expression of $\theta(t, I)$ is the same as (8)

$$
\theta(t, I)=\sum_{n=0}^{N-1} \operatorname{rect}\left(\frac{t-n \cdot T_{s}}{T_{s}}\right) \cdot \theta\left(t, I_{n}\right)
$$

And the phase of the $n$-th bit of MSK is expressed as

$$
\begin{aligned}
\theta\left(t, I_{n}\right)= & \frac{1}{2} \cdot \pi \cdot \sum_{k=-\infty}^{n-1} I_{k}+\pi \cdot I_{n} \\
& \cdot q\left(t-n \cdot T_{s}\right), \quad n \cdot T_{s} \leq t<(n+1) \cdot T_{s} .
\end{aligned}
$$

2.3. JRC Signal. In this paper, the MSK modulated data are embedded into NLFM signal to form the NLFM-MSK JRC signal. From the mathematical perspective, the NLFM signal is used as the carrier signal in MSK signal to form the novel JRC signal. The time-domain expression of our JRC signal is as follows:

$$
\begin{aligned}
s(t)= & s_{\mathrm{NLFM}}(t) \cdot s_{\mathrm{MSK}}(t)=A_{m} \cdot \operatorname{rect}\left(\frac{t}{T_{p}}\right) \\
& \cdot \exp [j \cdot(\phi(t)+\theta(t, I)+\varphi)] .
\end{aligned}
$$

Considering the design convenience, we assume $A_{m}=1$ and $\varphi=0$. Therefore, the final JRC signal is expressed as

$$
s(t)=\operatorname{rect}\left(\frac{t}{T_{p}}\right) \cdot \exp [j \cdot(\phi(t)+\theta(t, I))] .
$$

NLFM-MSK is characterized by a continuous phase. We assume $B=10 \mathrm{MHz}, T_{p}=20 \mu \mathrm{s}$, and the length of the modulated MSK sequences is 5, 10, and 20, respectively. JRC signal can carry data of $[-11-1-11],[-1-1-1-11-$ $111-1-1]$, and $[-1-11-1-11-111-11-1-1111$ $-1-111]$, which are pseudorandom sequences generated by the computer. The time-domain waveforms of the NLFM and JRC signals are shown in Figure 6. After the pseudorandom sequences are loaded, JRC signals have the characteristics of constant envelope and continuous phase. Additionally, the 


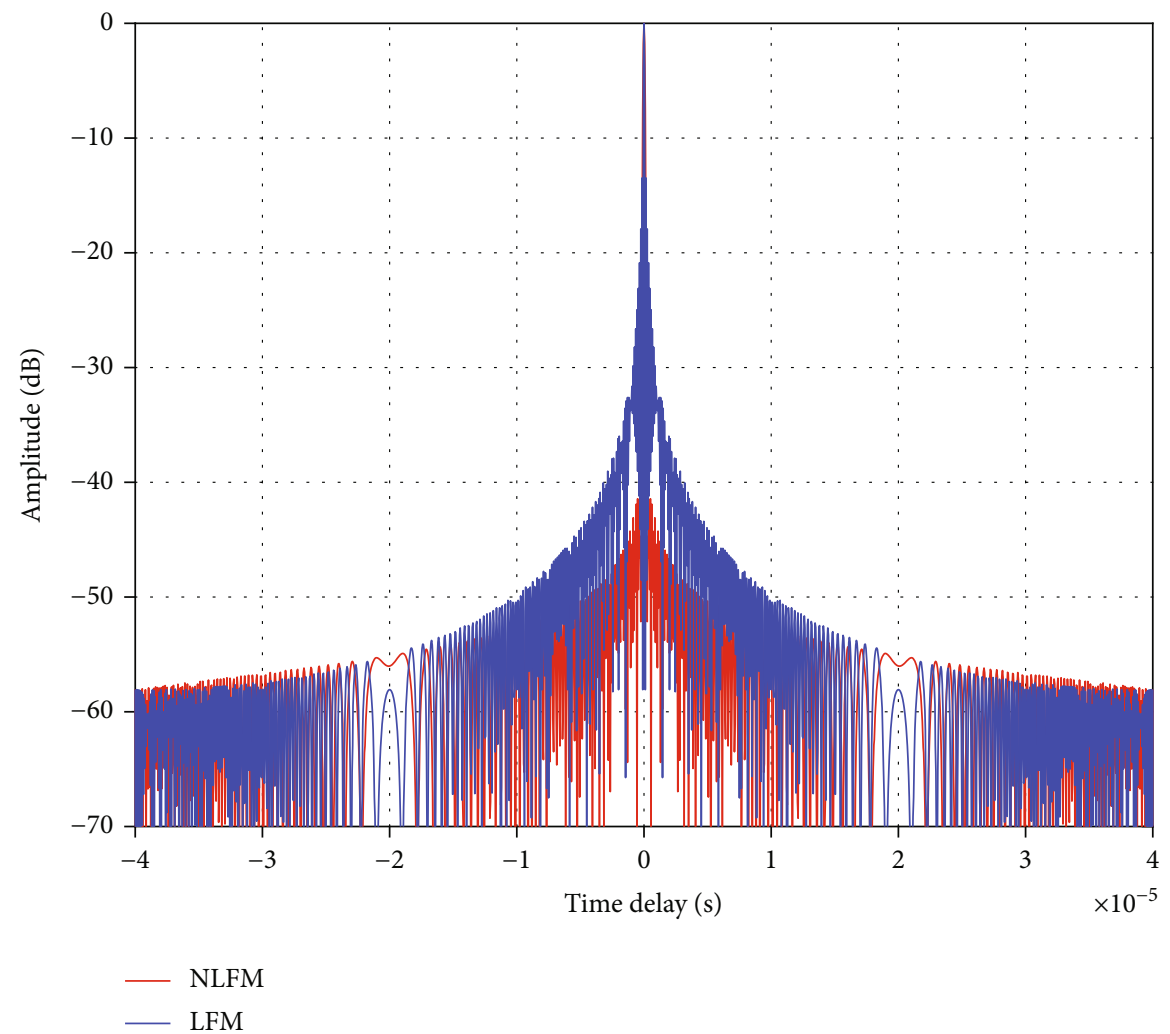

(a)

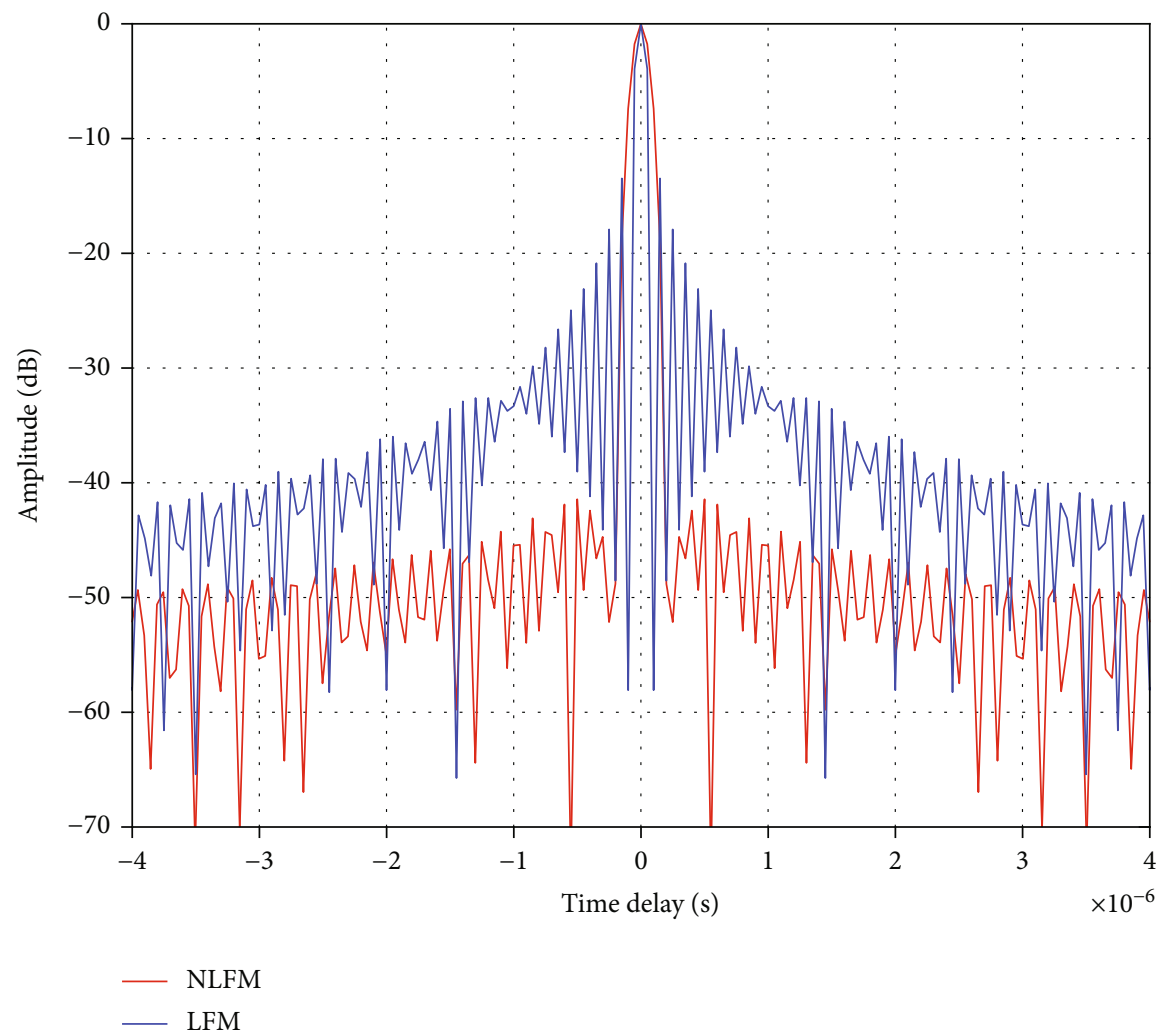

(b)

FIgURE 5: The autocorrelation of LFM and NLFM signals: (a) global chart; (b) local chart. 


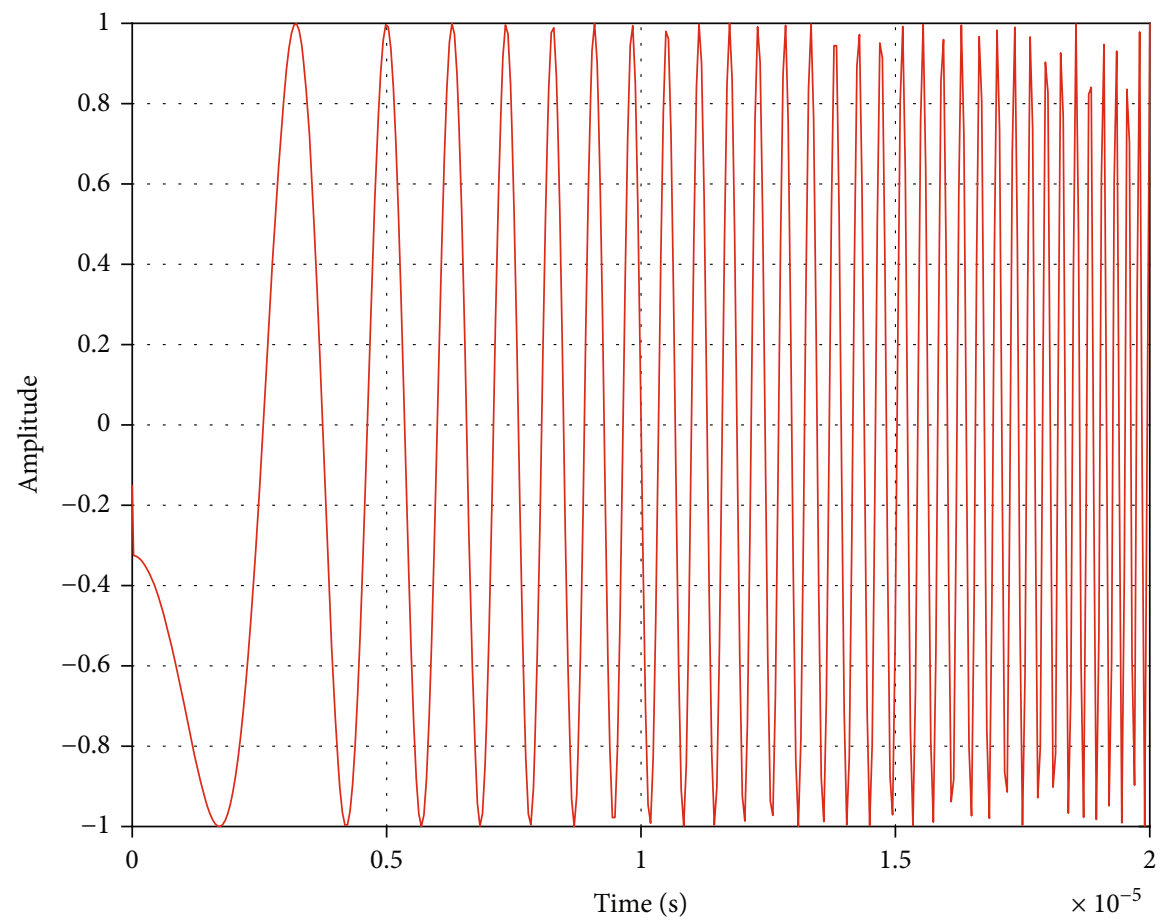

- NLFM

(a)

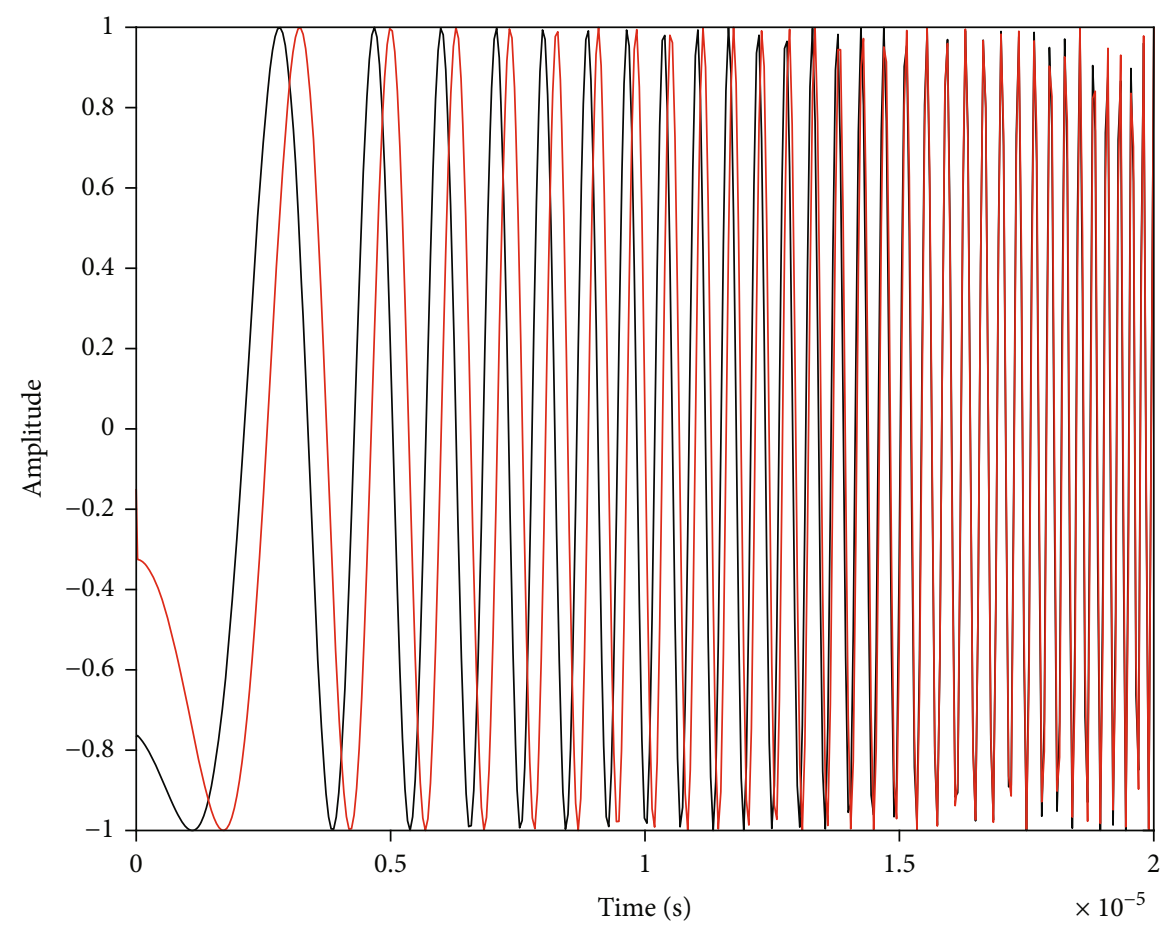

— JRC with 5 bits

- NLFM

(b)

FIgURe 6: Continued. 


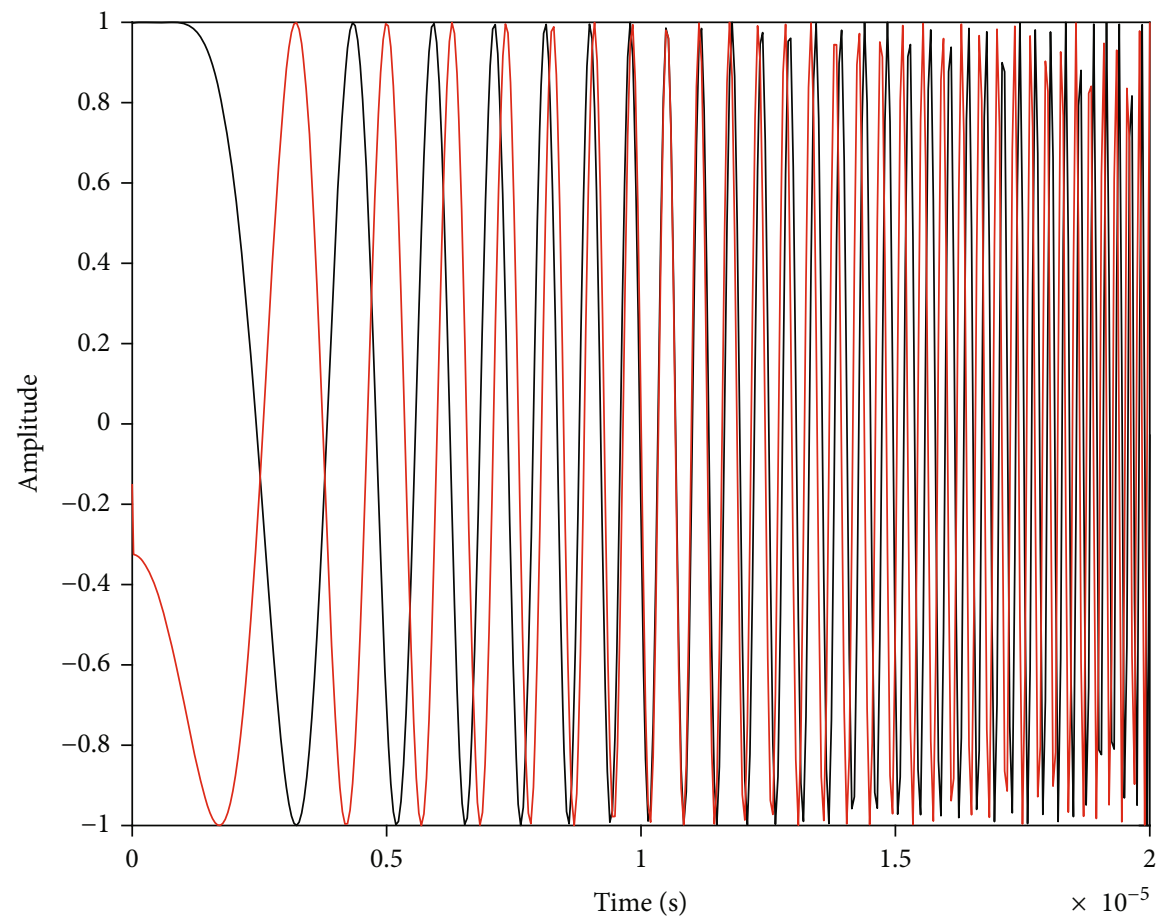

— JRC with 10 bits

- NLFM

(c)

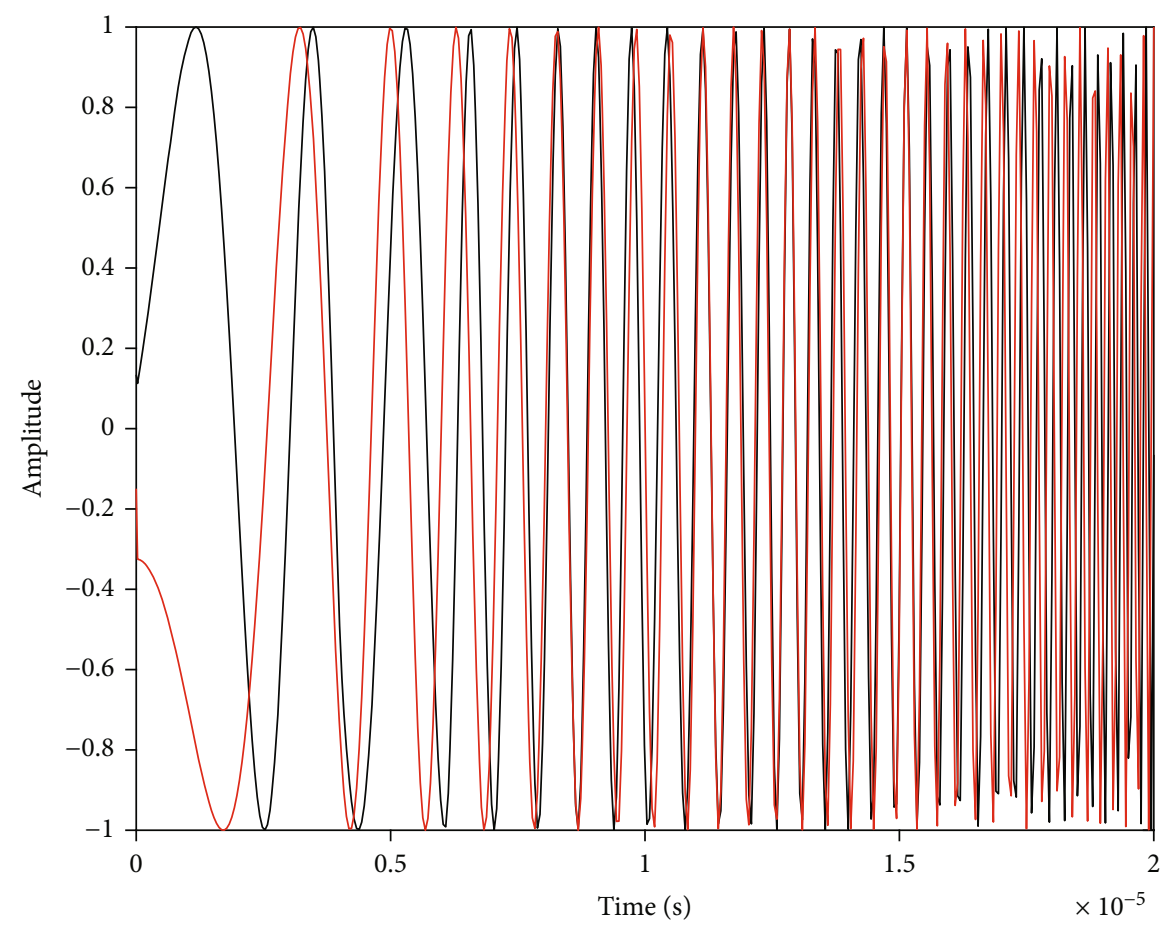

— JRC with 20 bits

- NLFM

(d)

FIGURE 6: The time-domain charts: (a) NLFM; (b) NLFM and JRC signal with 5 bits; (c) NLFM and JRC signal with 10 bits; (d) NLFM and JRC signal with 20 bits. 


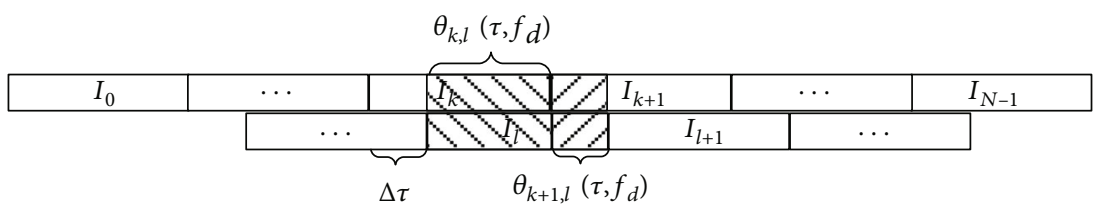

Figure 7: The diagram of numerical integration.

JRC signal guarantees the envelope characteristics of NLFM radar signal and the phase characteristics of MSK signal. Our JRC signal has excellent time-domain characteristics, which are continuous phase and constant envelope.

\section{Performance Analysis of JRC Signal}

JRC signal realizes radar detection and wireless communication simultaneously, and mutual interferences unavoidably take place. In order to evaluate the overall performances of JRC signal, the radar performance criteria and communication performance criteria are analyzed, respectively, in this section. In the paper, we focus on such criteria: AF for JRC, range resolution, detection probability, spectral characteristics, and communication rate of JRC signal. The corresponding mathematical equations have been carefully deduced and analyzed.

3.1. Performance Analysis of Radar. We first discuss the radar performance of the JRC signal. The radar waveforms mainly realize detecting and tracking the target and need to accomplish both an excellent detection accuracy and detection probability. By referring to literature [14, 29-32], the $\mathrm{AF}$ and detection probability can be used as the criteria of radar performance for our JRC signal. AF is one of the effective analysis tools in radar field. Time AF and Doppler AF can describe the range resolution and velocity resolution of radar waveform, respectively. An ideal radar AF should be a thumbtack-like characteristic, which is a peak point at the origin and zero elsewhere. But in practice, the radar signal is impossible to present a thumbtack shape. For radar signal, we hope that the time AF and Doppler AF have low sidelobe level, so the radar signal has perfect range resolution and velocity resolution. In this paper, the AF of JRC is defined as follows [33]:

$$
\chi\left(\tau, f_{d}\right)=\int_{0}^{T} s(t) \cdot s^{*}(t-\tau) \cdot e^{j \cdot 2 \cdot \pi \cdot f_{d} \cdot t} d t
$$

where $\tau$ and $f_{d}$ represent the time delay and Doppler shift of JRC signal echo. In literature $[12,13]$, the AF performances of LFM and LFM-MSK signals were analyzed and compared, and the range resolution and velocity resolution were analyzed in detail. Because there is no specific expression for the phase $\phi(t)$ in our JRC signal, it is impossible to derive the specific AF expression for the JRC signal in this paper. Considering the particularity of the integrated signal, the numerical integration method can be used to obtain the approximate value of the AF for JRC signal, and then, the range resolution and velocity resolution of our integrated signal can be analyzed through the time AF and Doppler AF.

We assume that JRC signal contains $N$ code elements, and the time width occupied by each code $I_{i}$ element is $T_{s}$, while $\Delta \tau$ represents the time delay of unit code $I_{i}$. The time delay of the JRC signal and the target echo signal is $\tau=(k$ $-l) \cdot T_{s}+\Delta \tau ; k, l=0,1,2, \cdots, N-1$. The numerical integration schematic diagram is shown in Figure 7 . We assume the above code elements are the JRC signal and the below code elements are the echo signal.

By substituting equation (14) into equation (15) and considering the numerical integration schematic, the $\mathrm{AF}$ expression of the JRC integrated signal can be obtained:

$$
\chi\left(\tau, f_{d}\right)=\sum_{l=0}^{N-k+l-1} \theta_{k, l}\left(\tau, f_{d}\right)+\sum_{l=0}^{N-(k+1)+l-1} \theta_{k+1, l}\left(\tau, f_{d}\right),
$$

where $\theta_{k, l}\left(\tau, f_{d}\right)$ is the cross-correlation function of the code slice $I_{k}$ and the code slice $I_{l}$ and $\theta_{k+1, l}\left(\tau, f_{d}\right)$ is the crosscorrelation function of the code slice $I_{k+1}$ and the code slice $I_{l}$. The JRC signal $s(t)$ with $N$ bits can be divided into $N$ parts, and $s_{k}(t)$ is the $k$-th part of JRC signal. As shown in Figure 7, $\theta_{k, l}\left(\tau, f_{d}\right)$ and $\theta_{k+1, l}\left(\tau, f_{d}\right)$ expressions are, respectively,

$$
\begin{aligned}
& \theta_{k, l}\left(\tau, f_{d}\right)=\int_{(k-l) \cdot T_{s}+\Delta \tau}^{(k-l+1) \cdot T_{s}} s_{k}(t) \cdot s_{l}^{*}(t-(k-l) \\
& \left.\cdot T_{s}-\Delta \tau\right) \cdot \exp \left(j \cdot 2 \cdot \pi \cdot f_{d} \cdot t\right) d t \\
& =\int_{(k-l) \cdot T_{s}+\Delta \tau}^{(k-l+1) \cdot T_{s}} \exp [\phi(t)-\phi(t-(k-l) \\
& \left.\cdot T_{s}-\Delta \tau\right)+\theta(t, I)-\theta((t-(k-l) \\
& \left.\left.\left.\cdot T_{s}-\Delta \tau\right), I\right)\right] \exp \left(j \cdot 2 \cdot \pi \cdot f_{d} \cdot t\right) d t \\
& =\int_{(k-l) \cdot T_{s}+\Delta \tau}^{(k-l+1) \cdot T_{s}} \exp [\phi(t)-\phi(t-(k-l) \\
& \left.\cdot T_{s}-\Delta \tau\right)+0.5 \cdot \pi \cdot \sum_{m=0}^{k-1} I_{m}+\pi \cdot I_{k} \\
& q\left(t-k \cdot T_{s}\right)-0.5 \cdot \pi \cdot \sum_{n=0}^{l-1} I_{n}-\pi \cdot I_{l} \\
& \cdot q\left(t-l \cdot T_{s}-(k-l) \cdot T_{s}-\Delta \tau\right)+2 \\
& \left.\cdot \pi \cdot f_{d} \cdot t\right] d t
\end{aligned}
$$




$$
\begin{aligned}
\theta_{k+1, l}\left(\tau, f_{d}\right)= & \int_{(k-l+1) \cdot T_{s}}^{(k-l+1) \cdot T_{s}+\Delta \tau} s_{k+1}(t) \cdot s_{l}^{*}(t-(k-l) \\
& \left.\cdot T_{s}-\Delta \tau\right) \cdot \exp \left(j \cdot 2 \cdot \pi \cdot f_{d} \cdot t\right) d t \\
= & \int_{(k-l) \cdot T_{s}+\Delta \tau}^{(k-l+1) \cdot T_{s}} \exp [\phi(t)-\phi(t-(k-l) \\
& \left.\cdot T_{s}-\Delta \tau\right)+\theta(t, I)-\theta((t-(k-l) \\
& \left.\left.\left.\cdot T_{s}-\Delta \tau\right), I\right)\right] \cdot \exp \left(j 2 \pi f_{d} t\right) d t \\
= & \int_{(k-l+1) \cdot T_{s}}^{(k-l+1) \cdot T_{s}+\Delta \tau} \exp [\phi(t)-\phi(t-(k-l) \\
& \left.\cdot T_{s}-\Delta \tau\right)+0.5 \cdot \pi \cdot \sum_{m=0}^{k} I_{m}+\pi \cdot I_{k+1} \\
& \cdot q\left(t-(k+1) \cdot T_{s}\right)-0.5 \cdot \pi \cdot \sum_{n=0}^{l-1} I_{n} \\
& -\pi \cdot I_{l} \cdot q\left(t-l \cdot T_{s}-(k-l) \cdot T_{s}-\Delta \tau\right) \\
& \left.+2 \cdot \pi \cdot f_{d} \cdot t\right] d t .
\end{aligned}
$$

Although the specific AF expression of JRC signal cannot been obtained, by observing equations (17) and (18), it can be found that due to the embedding communication data, the phase of JRC signal has a random shift, which will inevitably lead to the deterioration of the AF performance of JRC signal. If the degree of phase shift can be reduced, the AF performance of the integrated signal can be improved to some extent. As shown in Figure 7, when the data carried by the integrated signal is gradually reduced, the unit code gradually widens, and the ratio of time delay to the time width of the code element gradually approaches zero. At the moment $k, l$ and $N$ approach 1 , the correlation function of the JRC signal gradually approaches the correlation function of the original NLFM signal, and the AF of the JRC signal gradually approaches the AF of the NLFM signal, which is equivalent to the fact that the NLFM signal carries no communication data.

In the parameter setting of JRC signal, the appropriate number of codes is selected to make the time delay greater than zero and far less than the time width of codes, that is, the condition of $0 \leq \tau \ll T_{s}$ and $k=l$. In the analysis of equation (16) and Figure 7, the value of $\theta_{k+1, l}\left(\tau, f_{d}\right)$ is far less than the value of $\theta_{k, l}\left(\tau, f_{d}\right)$, and its influence on the AF can be ignored. From the perspective of analysis convenience, $\theta_{k, l}\left(\tau, f_{d}\right)$ can be rewritten as

$$
\begin{aligned}
\theta_{k, l}\left(\tau, f_{d}\right)= & \int_{\Delta \tau}^{T_{s}} s_{k}(t) \cdot s_{l}^{*}(t-\Delta \tau) \cdot \exp \left(j \cdot 2 \cdot \pi \cdot f_{d} \cdot t\right) d t \\
= & \int_{\Delta \tau}^{T_{s}} \exp [\phi(t)-\phi(t-\Delta \tau)+j \cdot 2 \cdot \pi \\
& \left.\cdot f_{d} \cdot t\right] d t, \text { while } 0 \leq \tau \ll T_{s}, k=l .
\end{aligned}
$$

Meanwhile, the influence of the second item in equation (16) can be ignored, and under the condition of $0 \leq \tau \ll T_{s}$,
TABLe 2: Simulation parameters.

\begin{tabular}{lc}
\hline Parameter & Value \\
\hline$T_{P}$ & $40 \mu \mathrm{s}$ \\
$B$ & $10 \mathrm{MHz}$ \\
$T_{s}$ & $T_{P} / N$ \\
$B T_{P}$ & 400 \\
$N$ & $5,10,20\left(N \ll B T_{P}\right)$ \\
$P_{f a}$ & $10^{-6}$ \\
PRI & $5 \mathrm{kHz}, 10 \mathrm{kHz}$ \\
Modulation & $\mathrm{MSK}$ \\
\hline
\end{tabular}

the AF for JRC signal can be approximately written as

$$
\chi\left(\tau, f_{d}\right)=\sum_{l=0}^{N-k+l-1} \theta_{k, l}\left(\tau, f_{d}\right) .
$$

The time AF of the JRC signal reflects the range resolution of the radar signal. We assume $f_{d}=0$ and derive time $\mathrm{AF}$ from equation (16), and then, time AF is expressed as

$$
\begin{aligned}
\chi(\tau, 0) & =\sum_{l=0}^{N-k+l-1} \theta_{k, l}(\tau, 0)=\int_{\Delta \tau}^{T_{p}} \exp [\phi(t)-\phi(t-\Delta \tau)] d t \\
& \approx \chi_{\mathrm{NLFM}}(\tau, 0) .
\end{aligned}
$$

From the analysis of equation (21), it can be seen that time AF of JRC signal is similar to the time AF of NLFM wave, and they have similar range resolution. For JRC signal, the conditions of $0 \leq \tau \ll T_{s}$ and $k=l$ should be guaranteed.

The Doppler AF of JRC signal reflects the velocity resolution of the radar signal. We assume $\tau=0$ and derive the Doppler AF equation; it can be rewritten as

$$
\begin{aligned}
\chi\left(0, f_{d}\right)= & \sum_{l=0}^{N-k+l-1} \theta_{k, l}\left(0, f_{d}\right)=T_{p} \cdot \exp \left(j \cdot \pi \cdot f_{d}\right. \\
& \left.\cdot T_{p}\right) \sin c\left(f_{d} \cdot T_{p}\right) \approx \chi_{\mathrm{NLFM}}\left(0, f_{d}\right) .
\end{aligned}
$$

Under the condition $0 \leq \tau \ll T_{s}$, JRC signal has the same velocity resolution as the original NLFM signal. Through the analysis of equations (15)-(22), although the exact expression of the JRC signal cannot be obtained, but its AF characteristics can be analyzed from the perspective of numerical analysis. Under the condition $0 \leq \tau \ll T_{s}$, the $\mathrm{AF}$, range resolution, and velocity resolution of the JRC signal are similar to the AF, range resolution, and velocity resolution of NLFM wave. When the number of data carried is increased, the condition $0 \leq \tau \ll T_{s}$ is not satisfied gradually, and the value of $\theta_{k+1, l}\left(\tau, f_{d}\right)$ cannot be ignored. The AF of JRC signal has greater fluctuation than the AF of NLFM, which leads to the deterioration of the performance of time AF and Doppler AF and affects the range resolution and velocity resolution. 
The detection probability of radar signal represents the probability of the echo signal plus the noise signal exceeding the threshold voltage, which is usually used to describe the ability for radar to detect targets. In this paper, the detection probability of JRC signal is considered as the radar performance criterion. The derivation process of detection probability is based on hypothesis testing [32, 33], which assumes that the working scenarios of the radar function of the integrated signal are divided into two scenarios. In the hypothesis testing, $H_{0}$ represents the working scenario with only noise, without target echo, while $H_{1}$ represents the working scenario where noise is mixed with the target echo. In the two detection scenarios, the received signals of JRC system are defined as $[33,34]$

$$
\begin{aligned}
& H_{0}: x(n)=\omega(n), \\
& H_{1}: x(n)=R+\omega(n),
\end{aligned}
$$

where $x(n)$ represents the received signal of the $n$-th sampling, $R$ represents the amplitude of echo signal, and $\omega(n)$ represents the additive white Gaussian noise with zero mean and variance $\sigma^{2}$.

In the detection scene $H_{0}$, the radar receiver receives only noise signals without echo signals, and the received signals are variables whose mean value is zero and variance is $\sigma^{2}$. The absolute value of the received signal $|x(n)|$ is the Rayleigh distribution signal, and the probability density function (PDF) of the signal is $[13,32]$

$$
f_{|x(n)|}\left(r \mid H_{0}\right)=\frac{2 \cdot r}{\sigma^{2}} \exp \left(-\frac{r^{2}}{\sigma^{2}}\right)
$$

where $r$ represents the amplitude of signal envelope. Assuming that the threshold voltage of detection is $V_{T}$, the expression of the false detection probability of the signal is $[14,33]$

$$
P_{f a}=\int_{V_{T}}^{\infty} f_{|x(n)|}\left(r \mid H_{0}\right) d r=\exp \left(-\frac{V_{T}^{2}}{\sigma^{2}}\right),
$$

and hence, detection threshold voltage can be expressed as

$$
V_{T}=\sqrt{\sigma^{2} \ln \left(\frac{1}{P_{f a}}\right)}
$$

In the hypothetical scenario $H_{1}$, the PDF of the signal envelope $|x(n)|$ is $[14,33]$

$$
f_{|x(n)|}\left(r \mid H_{1}\right)=\frac{2 \cdot r}{\sigma^{2}} \cdot \exp \left(-\frac{r^{2}+R^{2}}{\sigma^{2}}\right) \cdot I_{0}\left(\frac{2 \cdot r \cdot R}{\sigma^{2}}\right)
$$

In equation (27), function $I_{0}()$ is a first-order modified Bessel function. Detection probability is the maximum probability of signal detection target under the condition of determining false detection probability, and its expression is as follows $[14,33]$ :

$$
\begin{aligned}
P_{d}= & \int_{v_{T}}^{\infty} f_{|x(n)|}\left(r \mid H_{1}\right) d r=\int_{v_{T}}^{\infty} \frac{2 \cdot r}{\sigma^{2}} \\
& \cdot I_{0}\left(\frac{2 \cdot r \cdot R}{\sigma^{2}}\right) \cdot \exp \left(-\frac{r^{2}+R^{2}}{\sigma^{2}}\right) d r .
\end{aligned}
$$

Through formula derivation, the detection probability of radar can be rewritten as $[14,33]$

$$
P_{d}=Q\left[\sqrt{2 \cdot \mathrm{SNR}}, \sqrt{2 \cdot \ln \left(\frac{1}{P_{f a}}\right)}\right]
$$

where $Q$ is the Marcum function, SNR is the signal-tonoise ratio of the receiving end, and $P_{f a}$ is the false detection probability.

From the derivation of equation (29), it can be concluded that the JRC signal detection probability is directly related to the SNR of the receiver and the false detection probability and has nothing to do with other factors. If the SNR and false detection probability of the receiver remain unchanged, the integrated signal can be guaranteed to have the same detection probability under the condition of altering the carrier signal, modulation mode, and carrying communication data. Considering that our JRC signal is transmitted by the radar high-power transmitter, the energy attenuation of transmitting communication data is small, and the SNR of the radar receiver can be guaranteed to remain unchanged, so the detection probability can be guaranteed to remain unchanged, too. Therefore, the detection probability of the JRC signal is independent of the modulation mode and carrier signal, and its performance is equivalent to that of the seminal NLFM signal.

3.2. Performance Criteria of Communication. The communication performance criteria of JRC signal include BER, spectrum characteristics, and communication rate. According to the first literature $[12,13]$, from the point of the communication's view, the MSK-LFM signal and JRC signals can be considered as the MSK signal modulated by the carriers of LFM signal and NLFM signal, respectively. The BER of signal is related to the modulation mode; it can be deduced that MSK signal, LFM-MSK signal, and our JRC signal have the same BER in the case of ideal matched filter receiver, so the BER performance is not considered in this paper.

This paper analyzes the communication performance from the view of the spectrum characteristics and communication rate. The short-time Fourier transform (STFT) method is one of best suited for analyzing the spectrum performance $[12,13]$. In the STFT method, the given signal is divided into many tiny parts by the fixed sliding window. Because the tiny parts of signal in each window are almost stationary, the signal spectrum of each time period is derived 


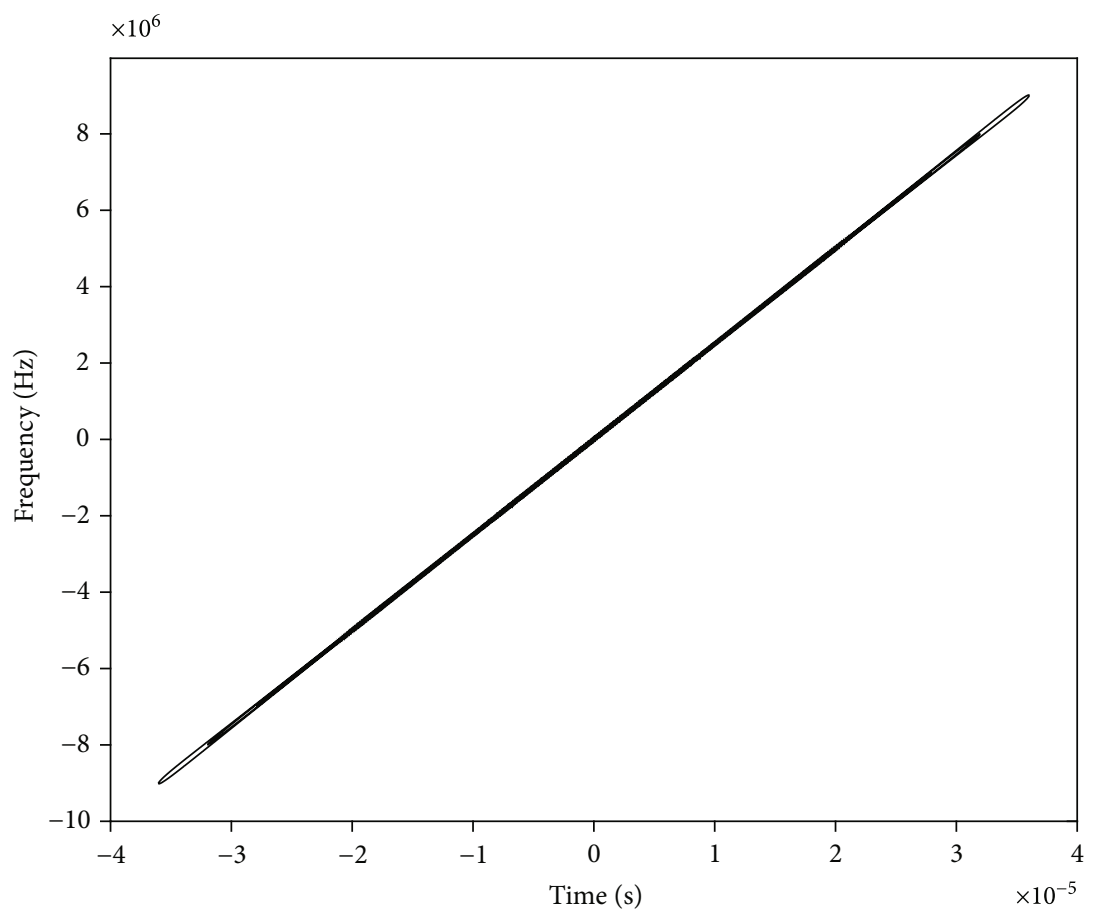

( $\mathrm{LFM}$

(a)

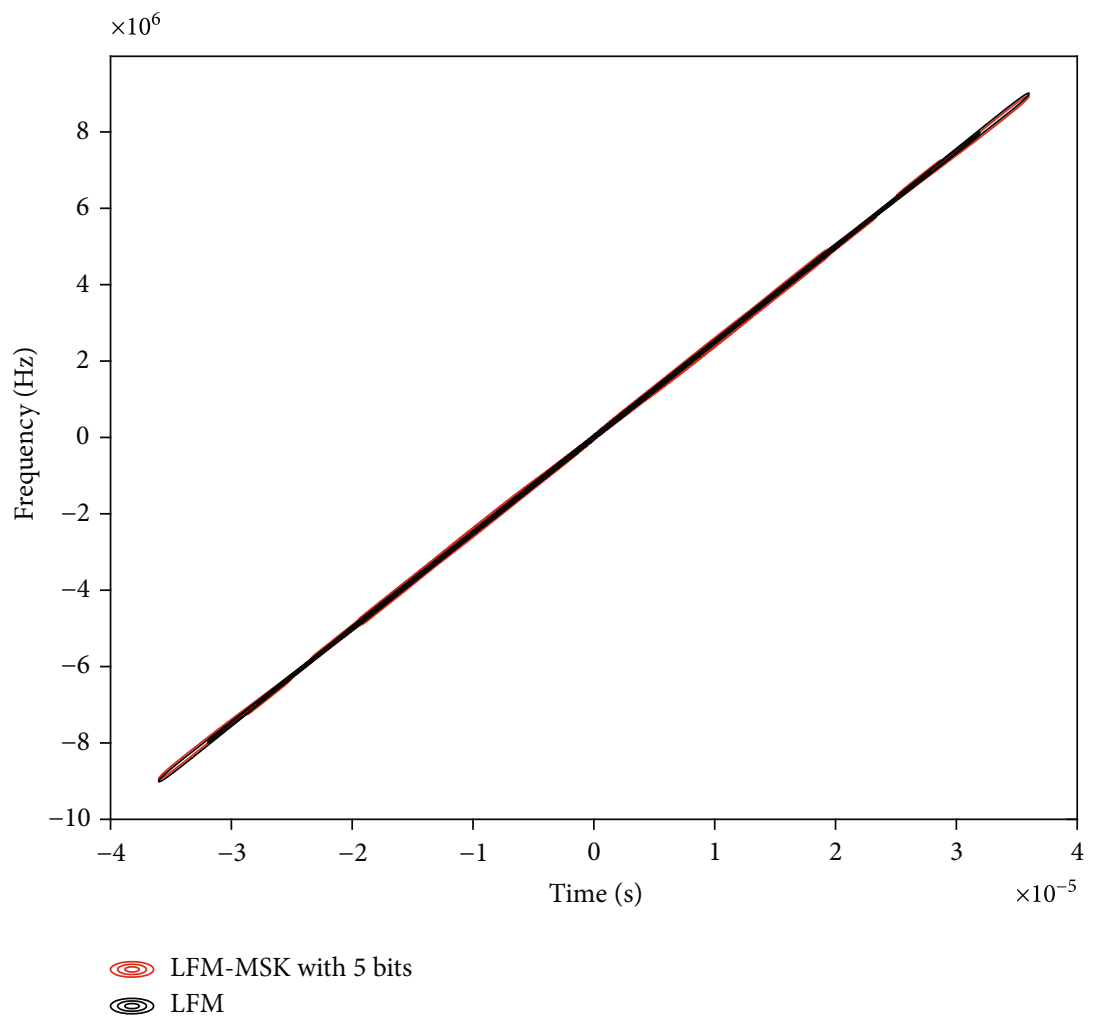

(b)

Figure 8: Continued. 


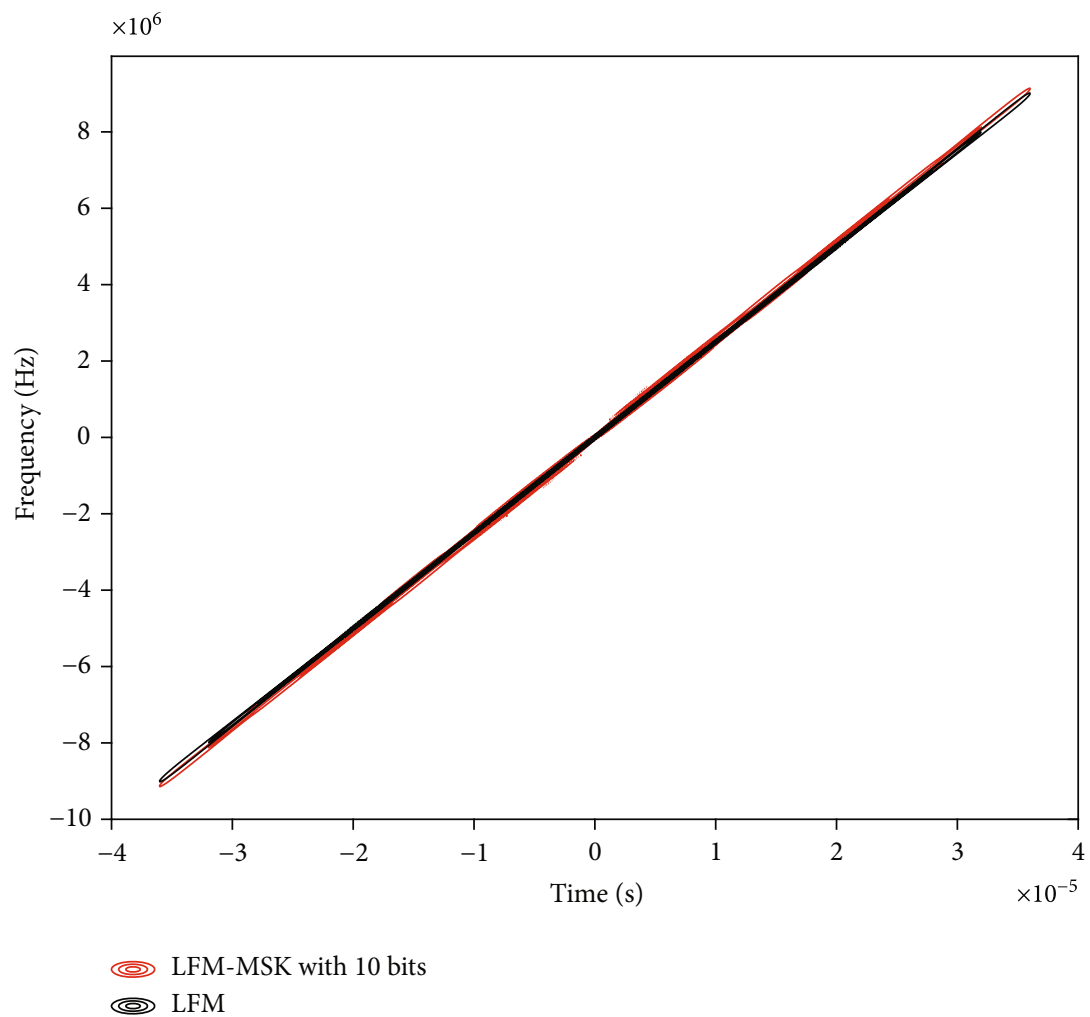

(c)

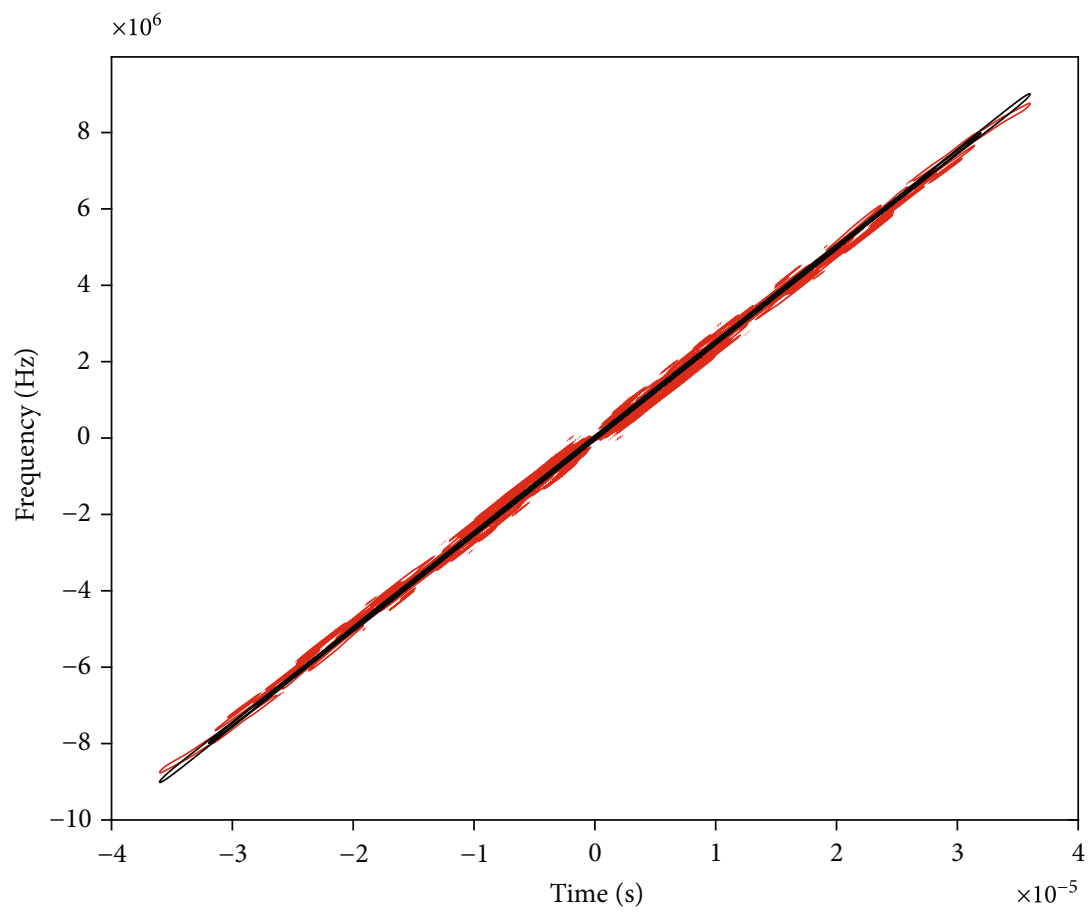

(ब) LFM-MSK with 20 bits

( LFM

(d)

FIGURE 8: Comparisons of top view AF of signals: (a) LFM; (b) LFM and LFM-MSK with 5 bits; (c) LFM and LFM-MSK with 10 bits; (d) LFM and LFM-MSK with 20 bits. 


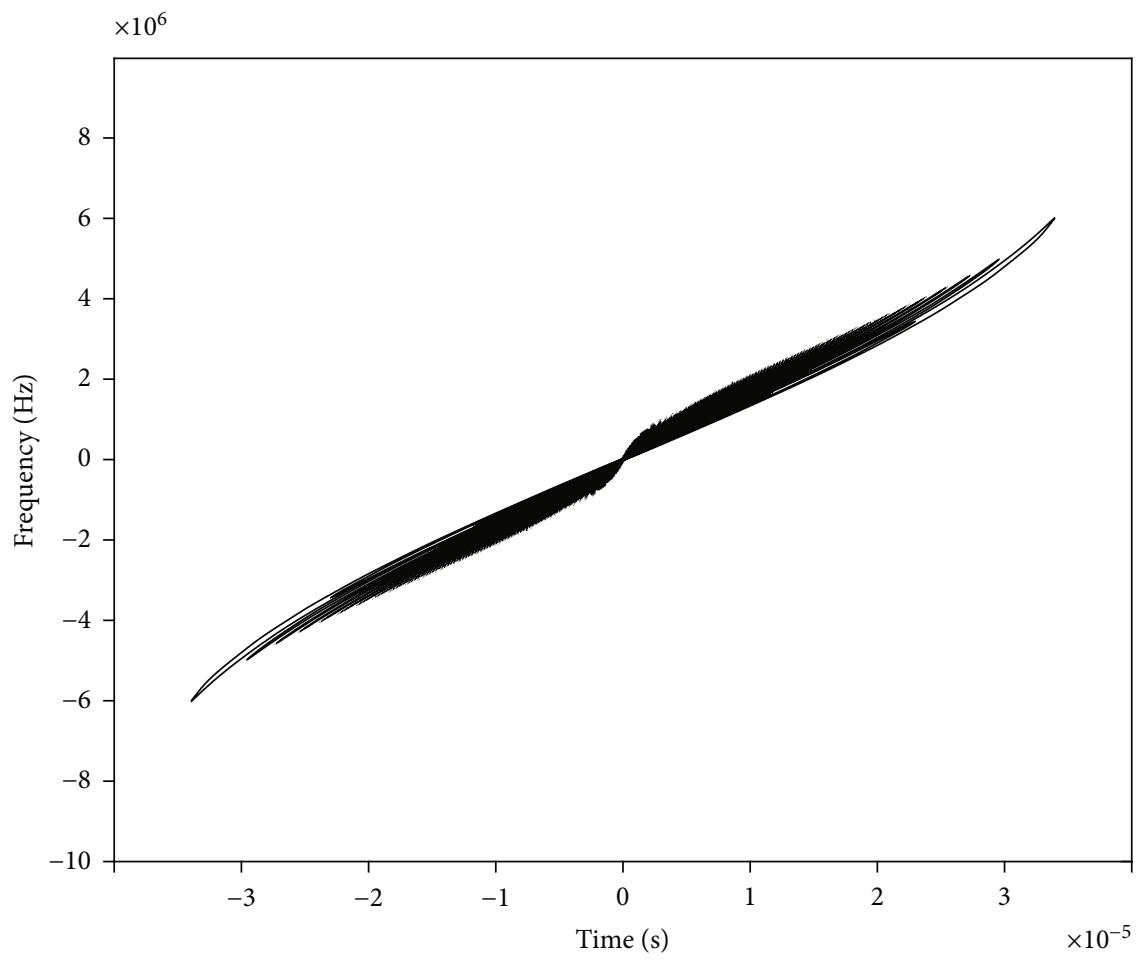

(NLFM

(a)

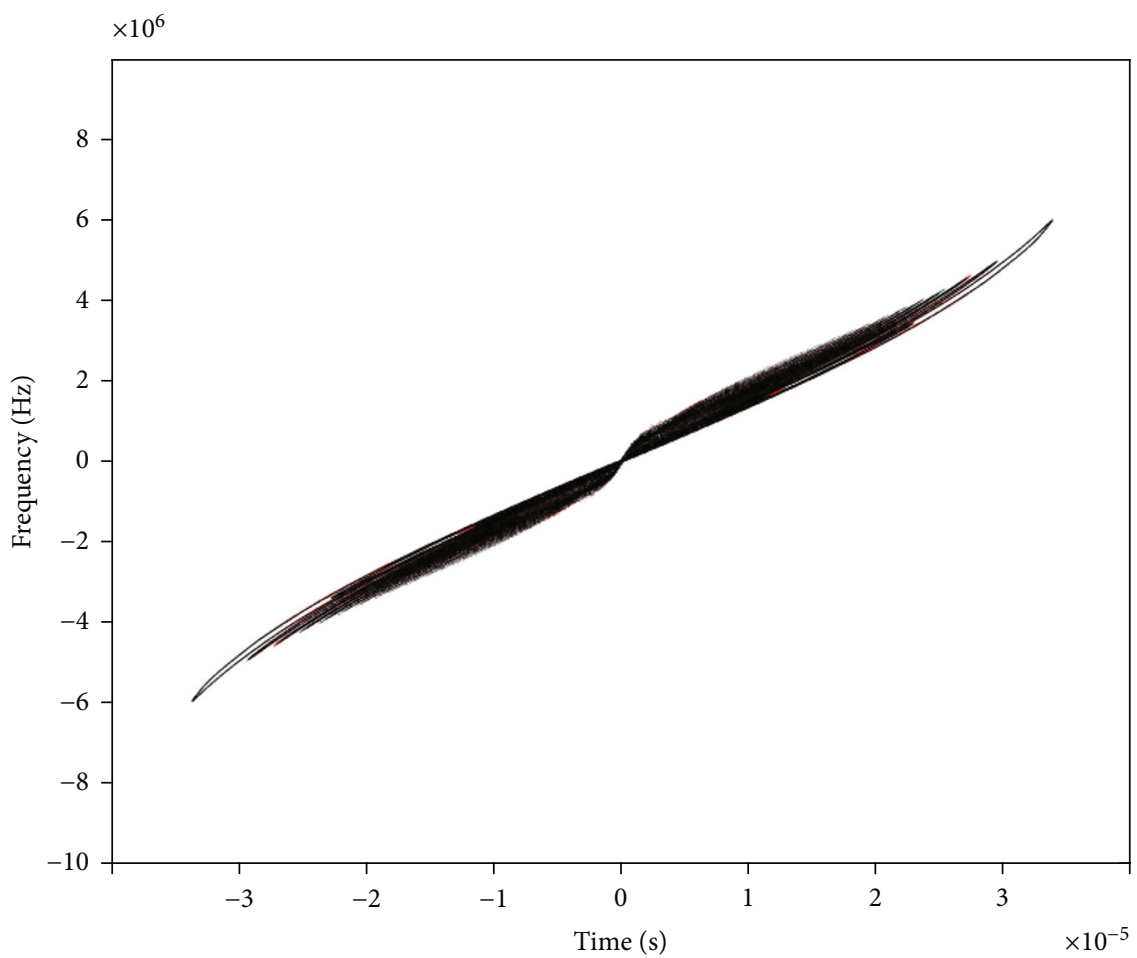

JRC with 5 bits

(a) NLFM

(b)

Figure 9: Continued. 


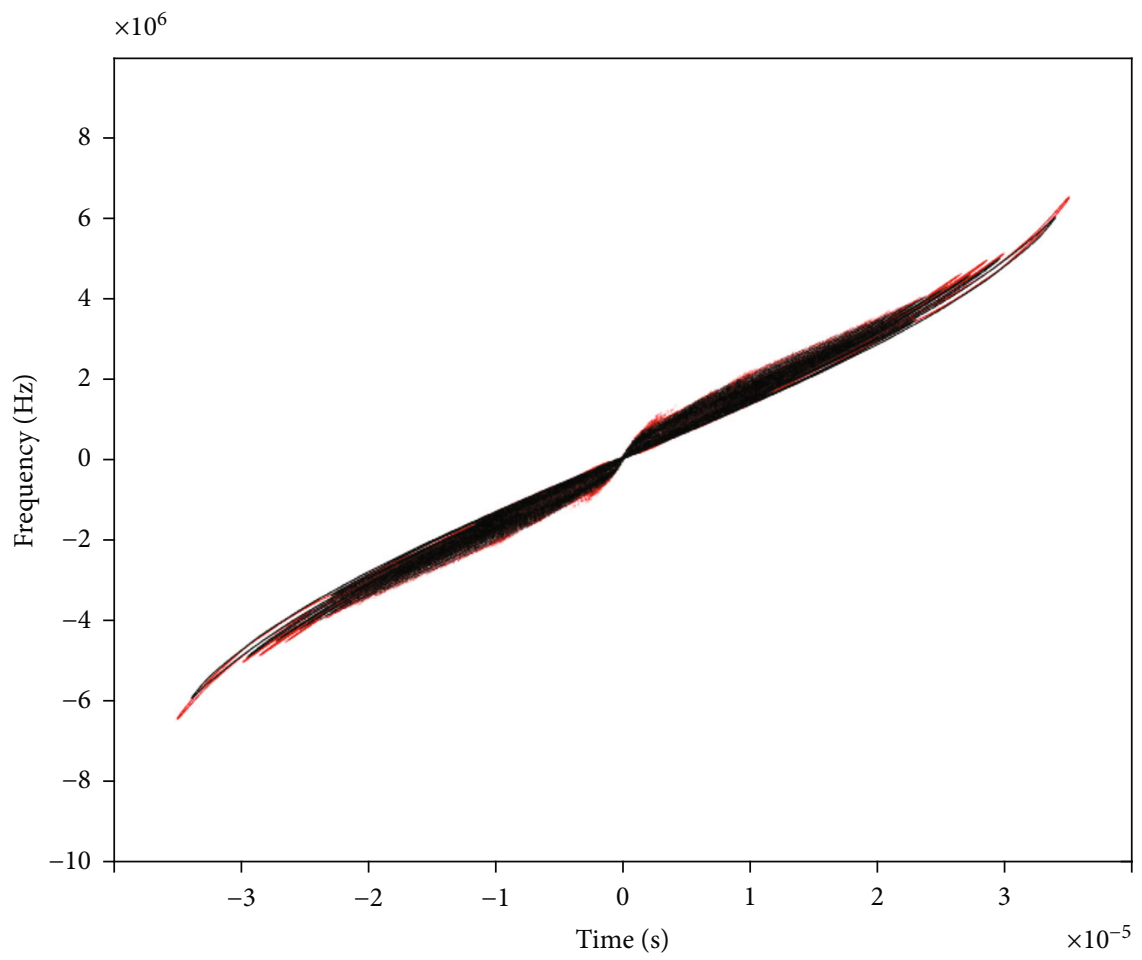

(6) JRC with 10 bits

( NLFM

(c)

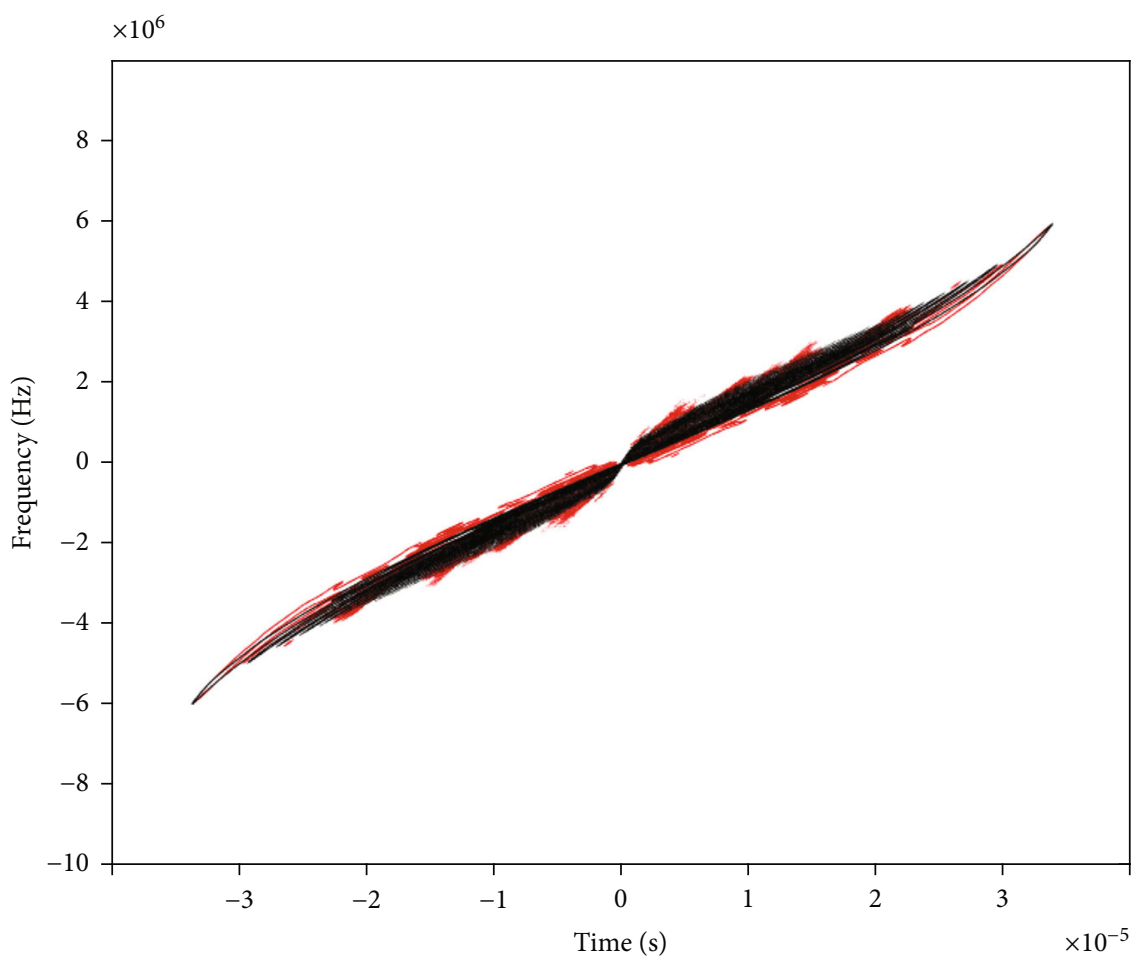

(a) JRC with 20 bits

(a) NLFM

(d)

Figure 9: Comparisons of top view AF of signals: (a) NLFM; (b) NLFM and JRC with 5 data; (c) NLFM and JRC with 10 data; (d) NLFM and JRC with 20 data. 


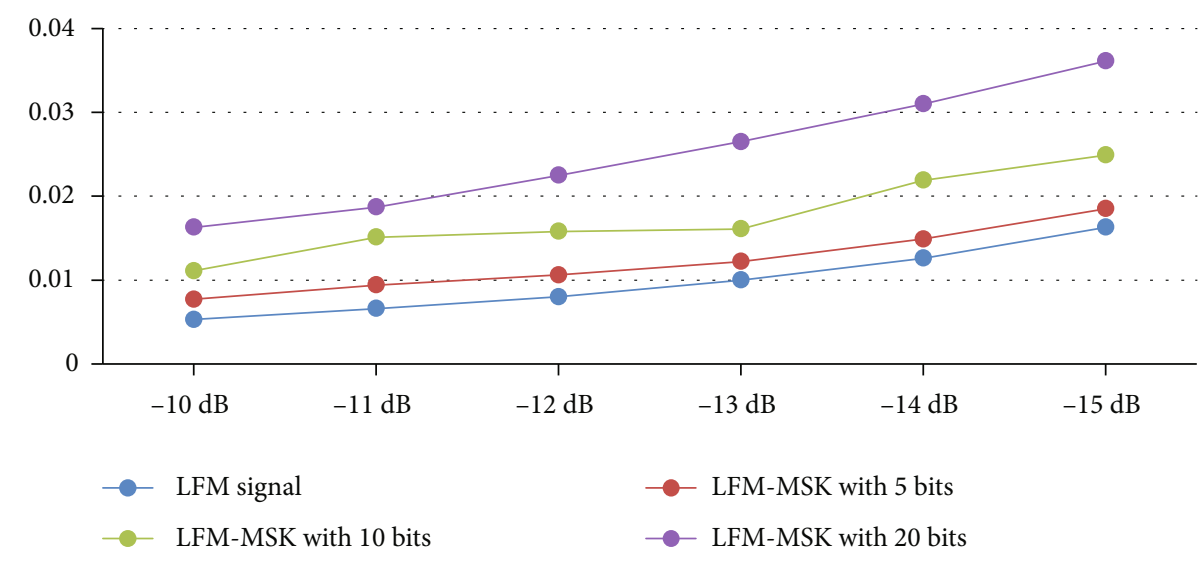

(a)

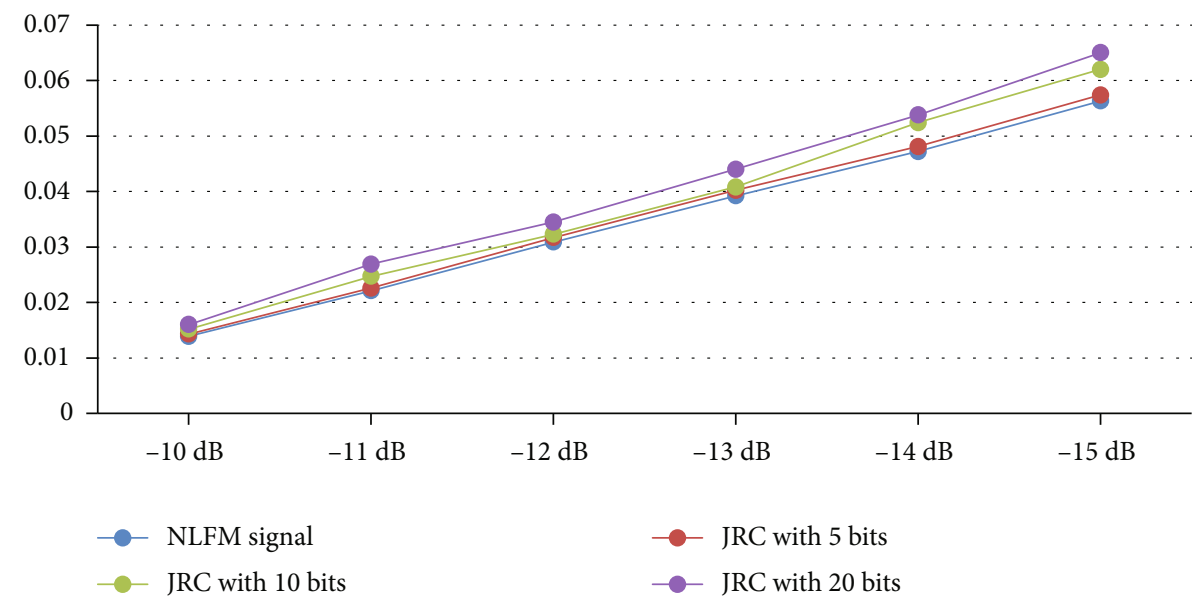

(b)

FIGURE 10: Density comparison of AF sparse matrixes for different threshold value: (a) LFM and LFM-MSK signals [12]; (b) NLFM and JRC signals.

through the Fourier transform. The STFT of the original signal $s(t)$ can be defined as follows:

$$
\operatorname{STFT}_{s}(t, f)=\int s(\tau) \cdot g^{*}(\tau-t) \cdot \exp (-j \cdot 2 \cdot \pi \cdot f \cdot t) d \tau
$$

We assume $g(t)$ is a real window function, and hence, $g^{*}(t)$ $=g(t)$. For the design convenience, we suppose that window function for our STFT transform is Gaussian window, whose size is 1024 , and its overlap is 1000 . We define that $S_{p}(t, f)$ is the spectrogram for signal $s(t)$ :

$$
S_{p}(t, f)=\left|\operatorname{STFT}_{s}(t, f)\right|^{2} .
$$

The energy distribution and communication characteristics can be described by signal spectrogram. For our JRC signal without precise equation, the exact mathematic expression of JRC signal's $S_{p}(t, f)$ and STFT function cannot be derived. Thanks to the former literature [12, 13], we can get the spectrogram for LFM-MSK and deduce some time-frequency characteristics for our JRC signal. Considering the amplitude, $S_{p}(t, f)$ for LFM-MSK signal is expressed as [12]

$$
S_{p}(t, f)=\exp \left\{\frac{2 \cdot\left[\pi \cdot \sigma \cdot\left(f-\left(u \cdot t+\sum_{i=1}^{N} u \cdot\left(t-i \cdot T_{b}\right) \cdot\left(p_{i} \cdot q_{i} / 4 \cdot T_{b}\right)\right)\right]^{2}\right.}{\left(2 \cdot \pi \cdot \sigma^{2} \cdot \mu\right)^{2}+1}\right\}
$$




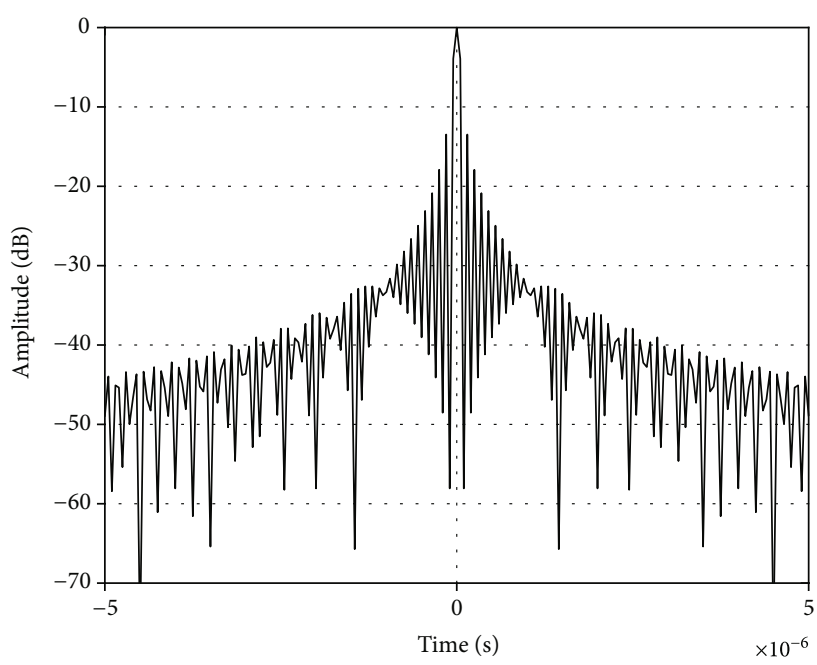

(a)

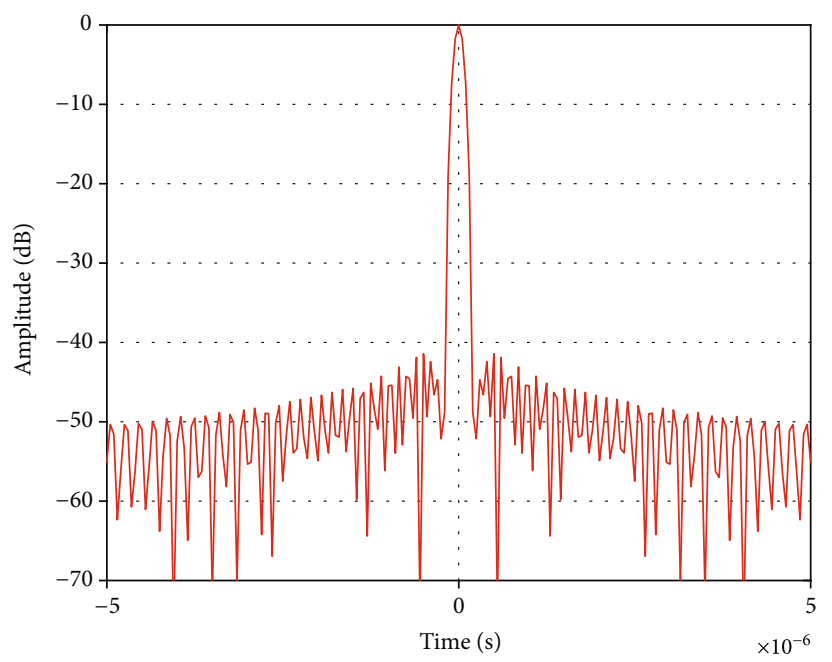

(c)

FIGURE 11: Comparisons of time AF of four signals: (a) LFM;

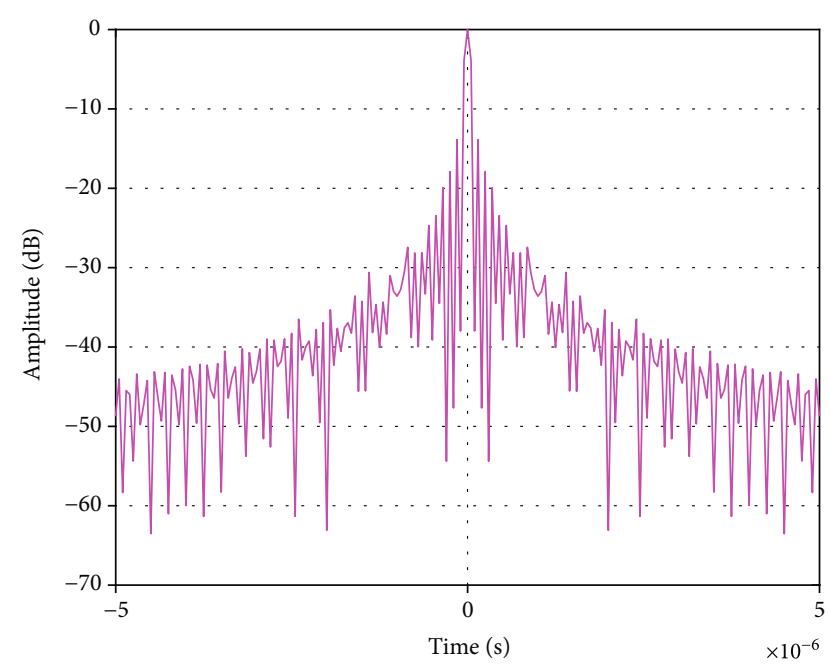

(b)

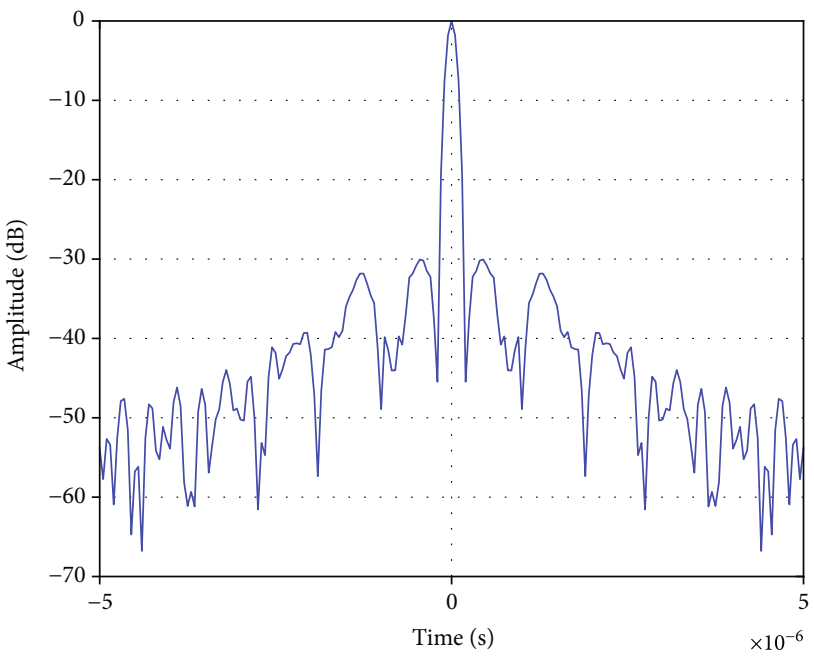

(d) where $p_{i}=\cos \left(\varphi_{i}\right), q_{i}=a_{i} \cos \left(\varphi_{i}\right), a_{i} \in\{ \pm 1\}$ is the $i$-th code for MSK, $\varphi_{i}$ is the initial phase of the $i$-th code, $u=B / T_{p}$, and $\sigma$ is constant. It is obvious that the spectrum of the LFMMSK waveform arranges along the line [12]

$$
u \cdot t+\sum_{i=1}^{N} u \cdot\left(t-i \cdot T_{b}\right) \cdot \frac{p_{i} \cdot q_{i}}{4 \cdot T_{b}}
$$

For the integrated LFM-MSK signal, with more communication data, the expansion accumulation of signal spectrum is more severe. The LFM-MSK signal with $N$ bits can be expressed as multiple sub-LFM signal. If $p_{i} \cdot q_{i}=1$, the spectrum of the $i$-th sub-LFM moves to $u \cdot t+\left(1 / 4 \cdot T_{b}\right)$; if $p_{i} \cdot q_{i}=-1$, the spectrum of the $i$-th sub-LFM moves to $u \cdot t$ $-\left(1 / 4 \cdot T_{b}\right)$. As the number of LFM-MSK symbols increases, and the energy distribution is more dispersed, and the cumulative effect of spectrum expansion is more obvious [12]. To reduce the spectrum spread for integrated signal, some methods for JRC signal can be considered such as car- rying less communication data, continuous phase modulation, and smart radar wave.

From equation (33), it can be deduced that if LFM-MSK does not carry data, in the time-frequency diagram the signal energy is linearly distributed along $u \cdot t$, which is the first derivative of phase for LFM signal. Here, assuming that the NLFM waveform does not carry data, the NLFM signal energy conforms to the curve distribution. Although explicit mathematics expression cannot be used, the NLFM signal distribution expression is used here to express the firstorder differential function of the original phase function, which is called $\phi^{\prime}(t)$. When our JRC carries $N$ bits of communication data, from the analogy of equation (33) and first literature $[12,13]$, we can know that our JRC signal energy distribution in time-frequency diagram is expressed as

$$
\phi^{\prime}(t)+\sum_{i=1}^{N} u \cdot\left(t-i \cdot T_{b}\right) \cdot \frac{p_{i} \cdot q_{i}}{4 \cdot T_{b}} .
$$




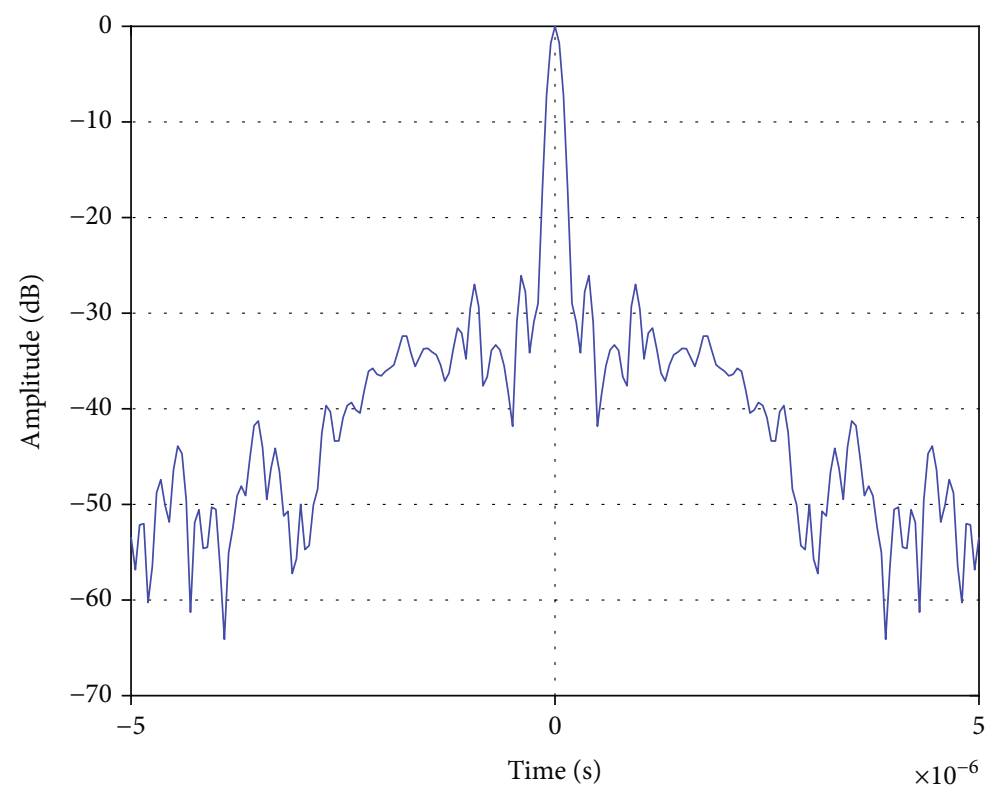

(a)

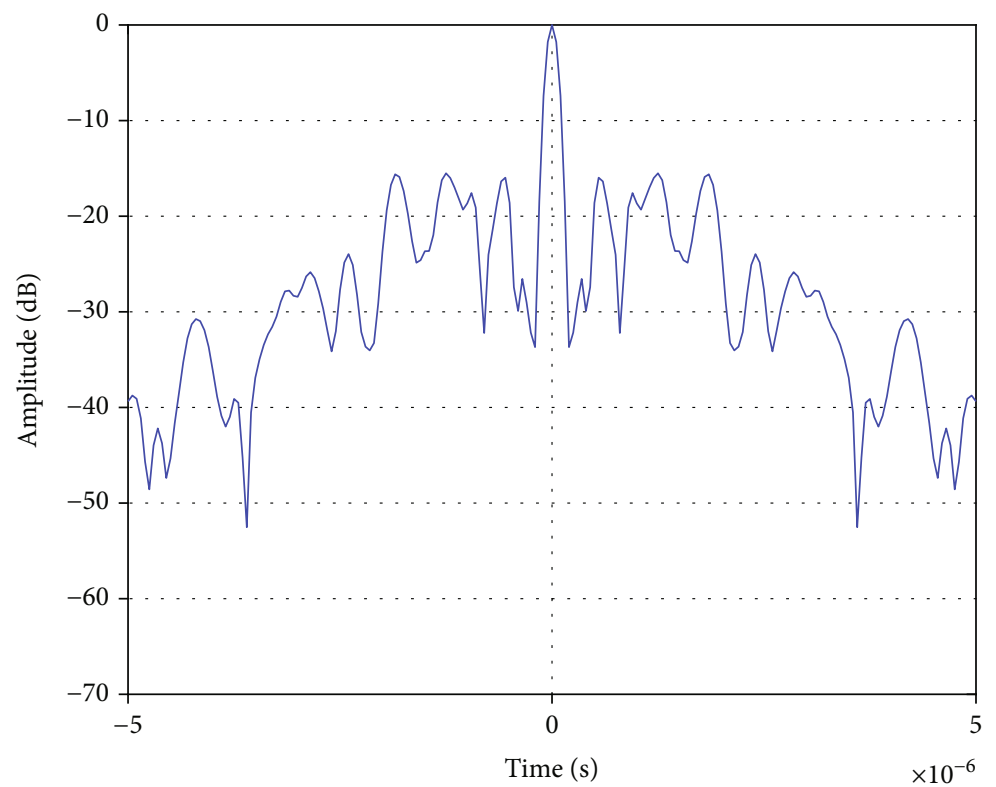

(b)

FIgURE 12: Comparisons of time AF of JRC with different data: (a) JRC with 10 data; (b) JRC with 20 data.

When the data change, its spectrum distribution is similar to that of LFM-MSK and has diffusion in different directions. If $p_{i} \cdot q_{i}=1$, the spectrum of the $i$-th sub-JRC moves to $\phi^{\prime}(t)+\left(1 / 4 \cdot T_{b}\right)$; if $p_{i} \cdot q_{i}=-1$, the spectrum of the $i$-th subJRC moves to $\phi^{\prime}(t)-\left(1 / 4 \cdot T_{b}\right)$.

If the communication data carried by JRC signal follow the condition $0 \leq \tau \ll T_{s}$, it can be deduced that the quantity of communication data should meet the following requirements:

$$
\begin{aligned}
& 0 \leq \tau \ll T_{s}=\frac{T_{P}}{N}, \\
& 0 \leq N \ll T_{P} / \tau=B \cdot T_{P} .
\end{aligned}
$$

In other words, the amount of communication data must be much less than the time bandwidth product in our JRC signal. Compared with the seminal NLFM signal, the spectrum spread caused by JRC signal is within the tolerable range due to the small amount of carried data, and the energy leakage of the integrated signal is effectively suppressed. In addition, the MSK modulation mode has the characteristics of phase continuity, so JRC signal has a small degree of spectrum expansion and almost the same spectrum bandwidth as the initial signal. In one word, under the condition $0 \leq N \ll B \cdot T_{P}$ and the MSK modulation, the energy shift of JRC spectrogram is not obvious, and it has good spectrum characteristics. 


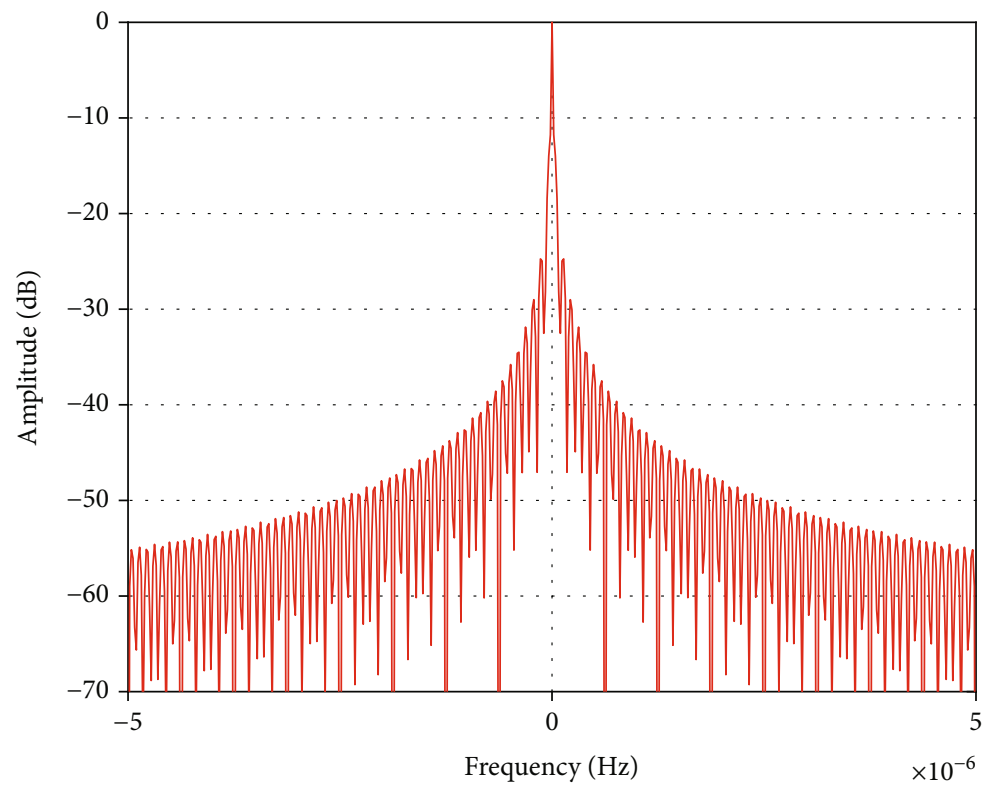

(a)

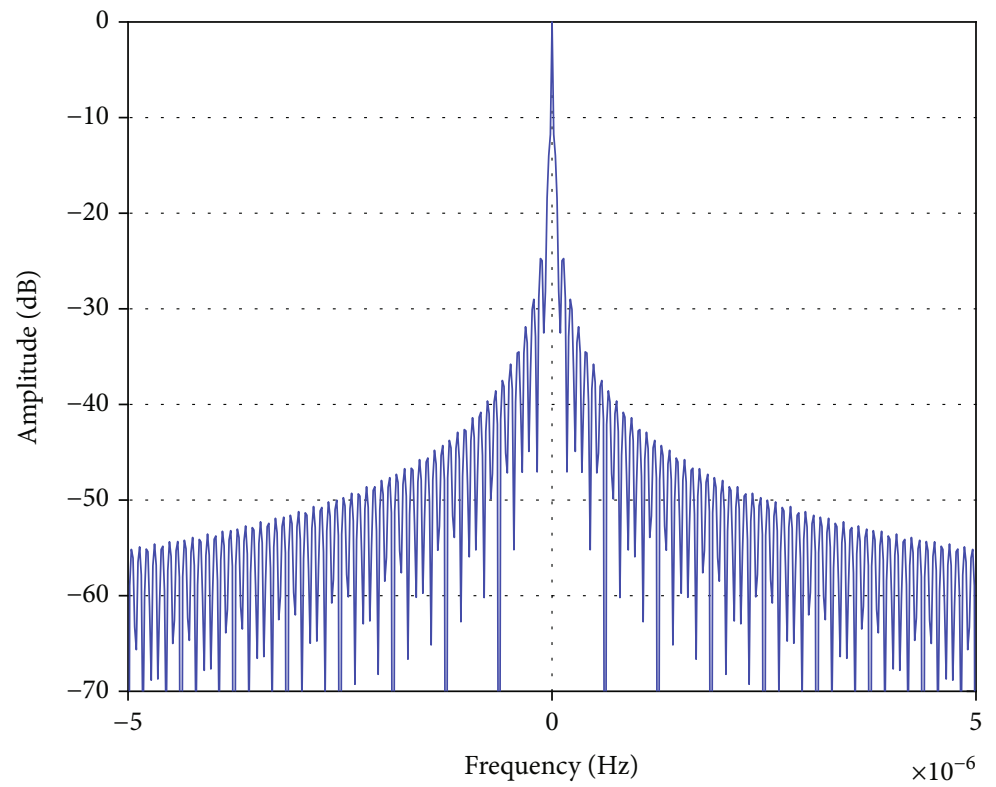

(b)

Figure 13: Continued. 


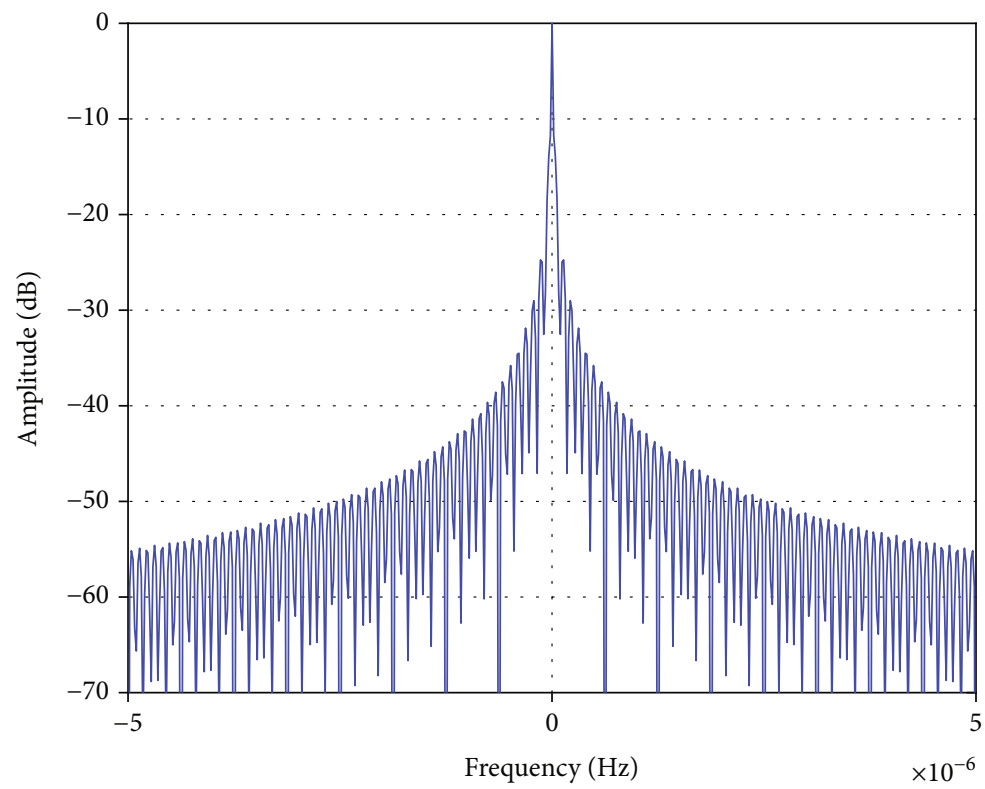

(c)

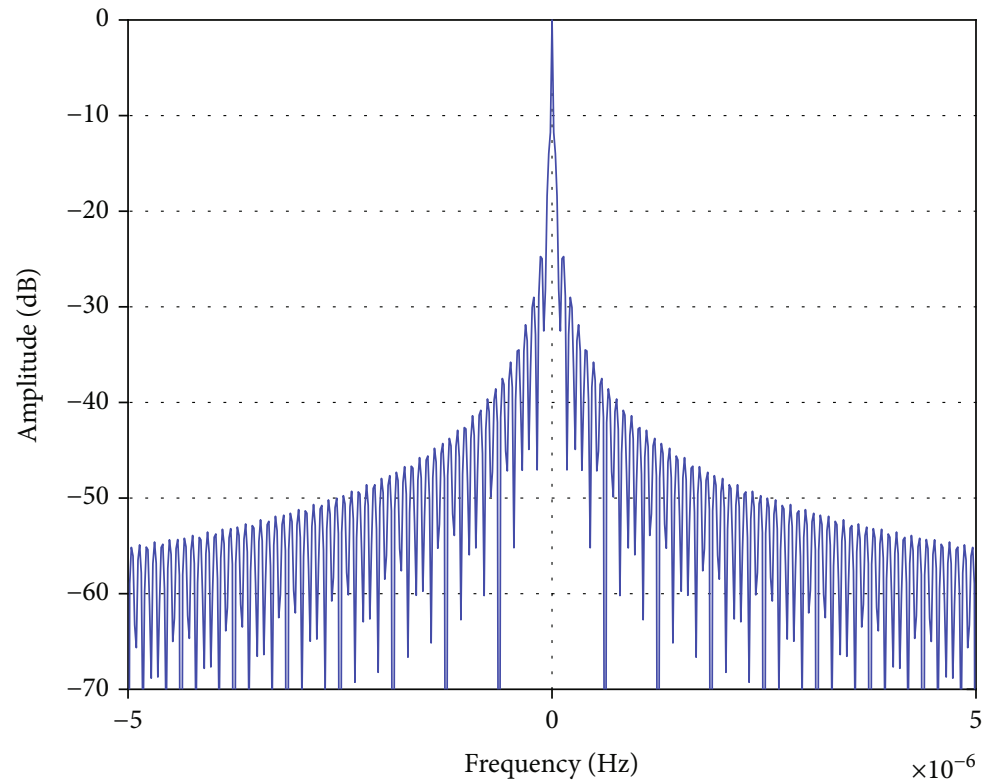

(d)

FIgure 13: Comparisons of Doppler AF of four signals: (a) NLFM; (b) JRC with 5 bits; (c) JRC with 10 bits; (d) JRC with 20 bits.

Communication rate is another important communication performance of JRC signal, which is related to unit pulse data carrying amount, pulse repetition frequency (PRI), and time width. We assume that the time width of the seminal NLFM pulse signal is $40 \mu$ s and the data carried is 5 bits. When the PRI is $1 \mathrm{kHz}$, the communication rate is $5 \mathrm{kHz} / \mathrm{s}$. Accordingly, when the PRI is $10 \mathrm{kHz}$, the communication rate is $50 \mathrm{kHz} / \mathrm{s}$.

In short, under the condition $0 \leq N \ll B \cdot T_{P}$, JRC signal designed in this paper has excellent detection performance and detection probability, and JRC signal spectrum spread is small. By setting appropriate parameters, it can have a high communication rate. The simulation results in Section
4 verify the theoretical derivation and performance analysis in this section.

\section{Simulation and Discussions}

In this section, we compare by means of numerical simulations the performance of our JRC signal with LFM-MSK signal [12] and seminal NLFM signal. The simulation results show our JRC signal can improve the range detecting performance for the same parameters. The parameter settings of JRC signal are listed in Table 2 . For the condition $0 \leq N \ll$ $B \cdot T_{P}$, we assume JRC signal carries 5,10 , and 20 bits of binary communication data, and the parameter $N$ is not 


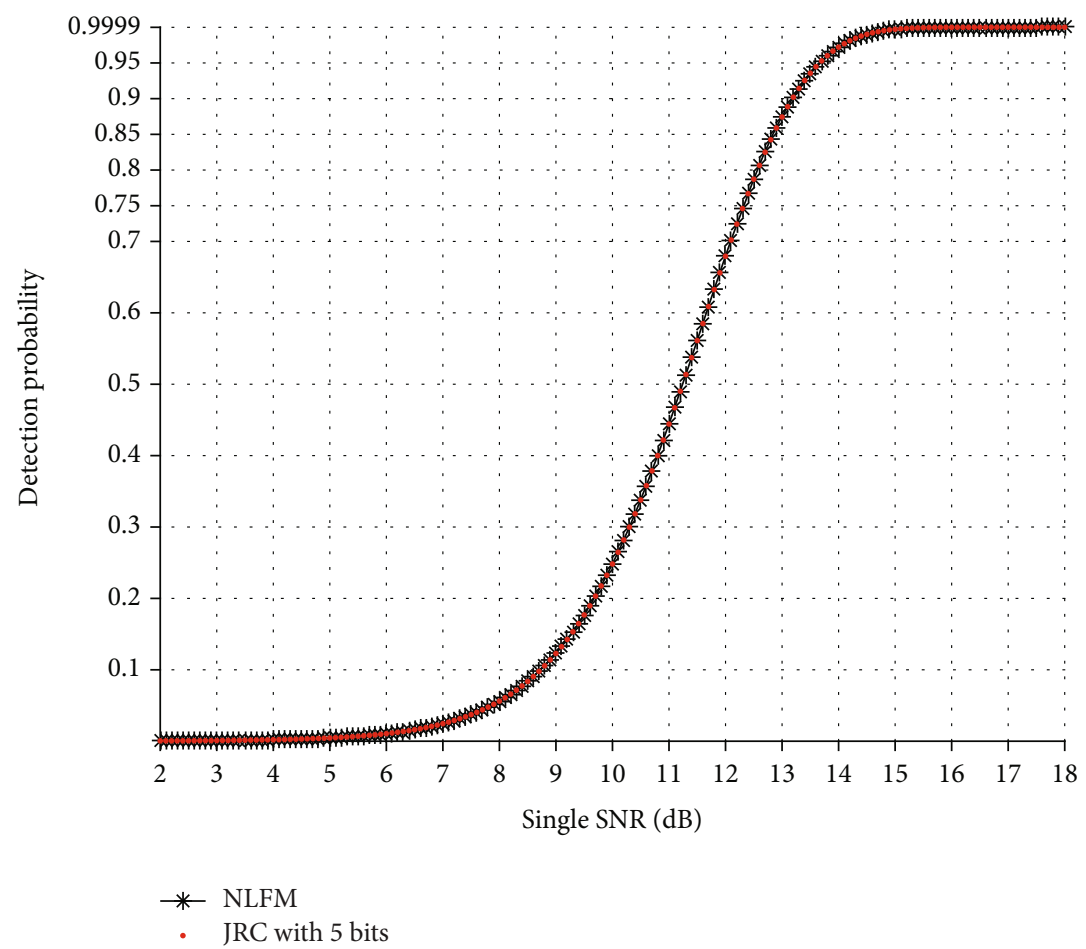

FIgURE 14: Detection probability diagram of JRC with 5 bits and NLFM.

larger than 20. In literature [12], LFM-MSK was used for integrated signal design, and the symbol pulse function of MSK is a rectangular function. Under the same condition, radar performance of the LFM-MSK signal and our JRC signal is compared to verify the performance analysis results in Section 3. We assume all the simulation channels are AWGN channels and the system are synchronized all the time.

4.1. Radar Performance Simulation. We compare by means of AF, time AF, and Doppler AF of four signals, which are LFM signal, NLFM signal, LFM-MSK signal [12], and our JRC signal. The top view AF of the LMF and LMF-MSK signals are shown in Figure 8. As shown in Figure 8, the AF of original LFM signal is in the shape of an oblique blade. In order to observe the spreading effect of LFM-MSK, we simulate the top view of the AF for LMF-MSK with different bits, compared with the top view of AF for seminal LFM signal. On this LFM basis, the performance of the AF of LFMMSK signal deteriorates due to the embedded communication information; with the more communication bits, the AF spreads more obviously.

As shown in Figure 9, the top view AF of the seminal NLFM and JRC signals are compared. Firstly, the top view AF of NLFM signal shows a curve feature, and JRC signal designed in this paper inherits the characteristics of NLFM signal, i.e., its AF has the same shape of such a curve itself. Secondly, with more communication data for JRC signal, the diffusion effect of the AF is more obvious. While the communication function is realized, the detection accuracy is sacrificed.

Because the AF of LFM-MSK signal [12] is extremely complicated and the AF of JRC signal has no specific expression, it is impossible to compare the AF mathematic expres- sions for LFM-MSK and JRC signals. And the spreading effect of AF of the JRC signal and LFM-MSK signal [12] is hard to deduce. But through the matrix's viewpoint and numerical simulation, we can compare the spreading effect of AF for LFM-MSK [12] and JRC. In the paper, most elements of the AF matrixes are far less than the $-10 \mathrm{~dB}$ of top AF element (threshold value), and the elements less than threshold value are approximately zero. We can set the threshold value from $-10 \mathrm{~dB}$ to the $-15 \mathrm{~dB}$ of top $\mathrm{AF}$ element value in the simulation. The AF matrixes of LFM, LFMMSK, NLFM, and JRC signals are all approximative sparse matrixes, as shown in Figures 8 and 9. If most of the elements of a matrix have 0 value, then it is called a sparse matrix; the density of a sparse matrix is the number of nonzero elements divided by the total number of matrix elements [35]. We assume the AF matrixes of LFM and NLFM signals are the initial sparse matrixes, and the AF matrixes of the LFM-MSK and JRC signals are the integrated sparse matrixes. Through the density calculation of the integrated sparse matrixes and the initial sparse matrixes, we can judge the degree of diffusion of sparse matrix and judge the spreading effect of AFs. In this paper, under the condition $0 \leq N \ll B \cdot T_{P}$, the bits carried by LFM-MSK [12] and JRC are 5,10 , and 20 , respectively, and the density simulations of the integrated sparse matrixes are carried out. As shown in Figure 10, the density of integrated sparse matrixes for LFM-MSK [12] and JRC signal can express the spreading effect. For the same parameters, JRC signal will inevitably spread after carrying the communication data, but the spreading effect is smaller than that of the LFM-MSK wave, which is shown in Figures 8-10. So our JRC's radar AF performance is better in the low data volume condition. 


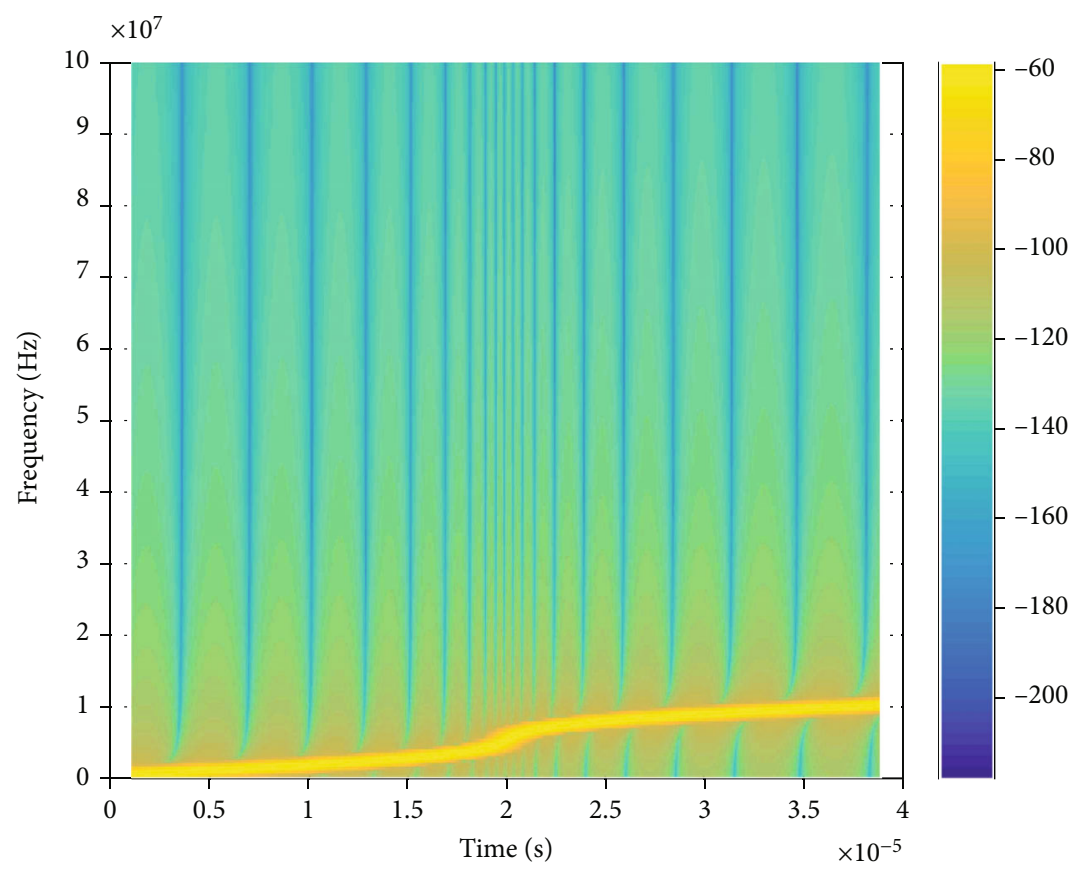

(a)

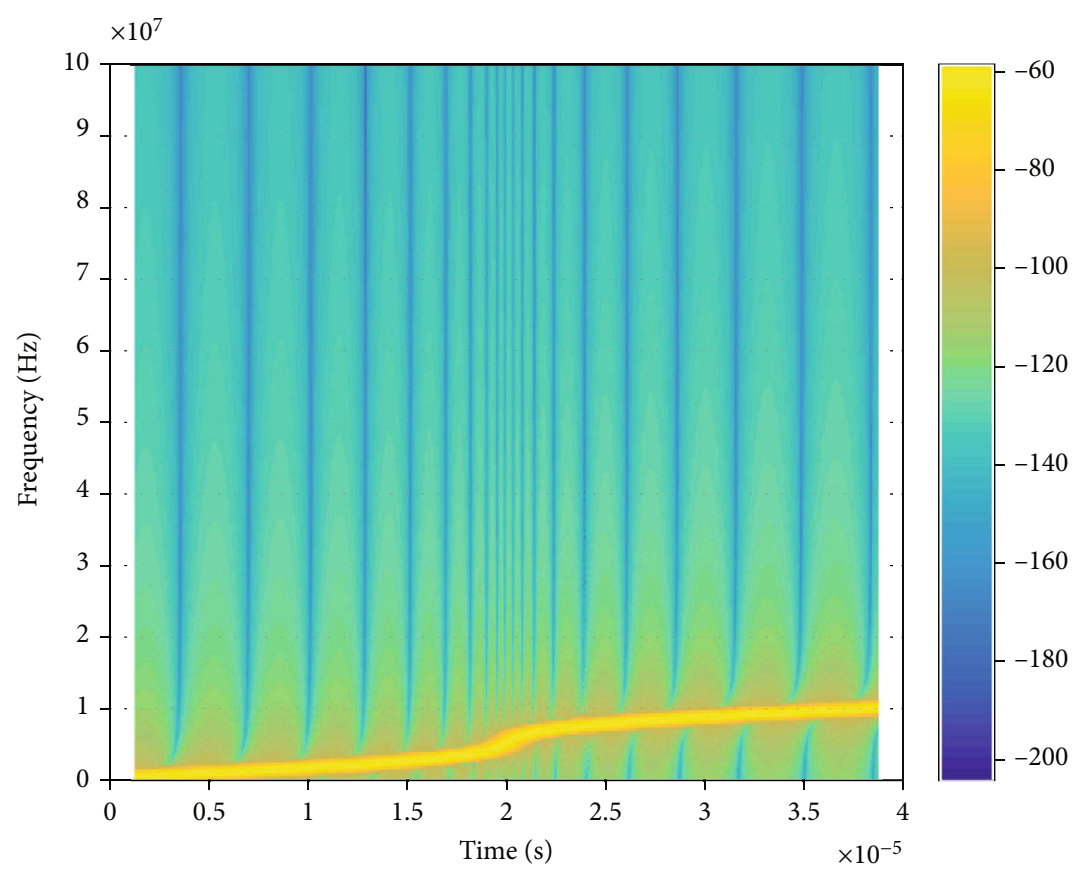

(b)

Figure 15: Continued. 


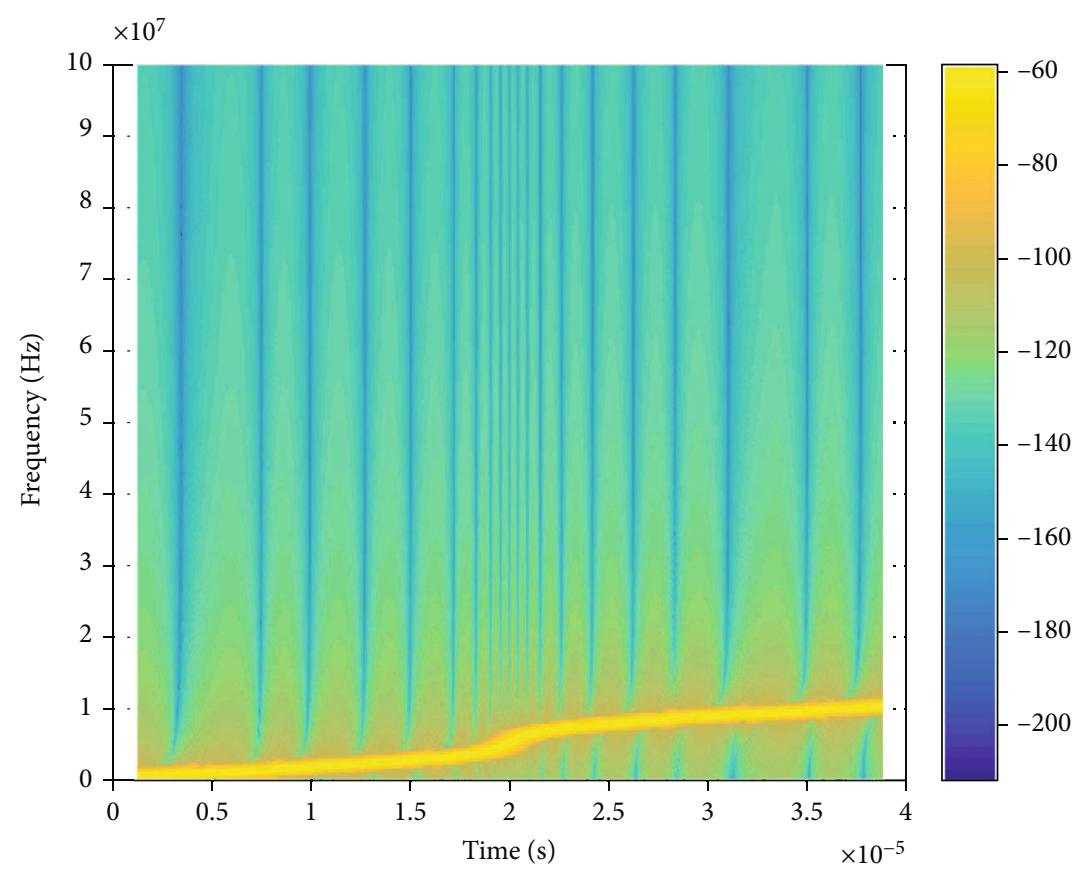

(c)

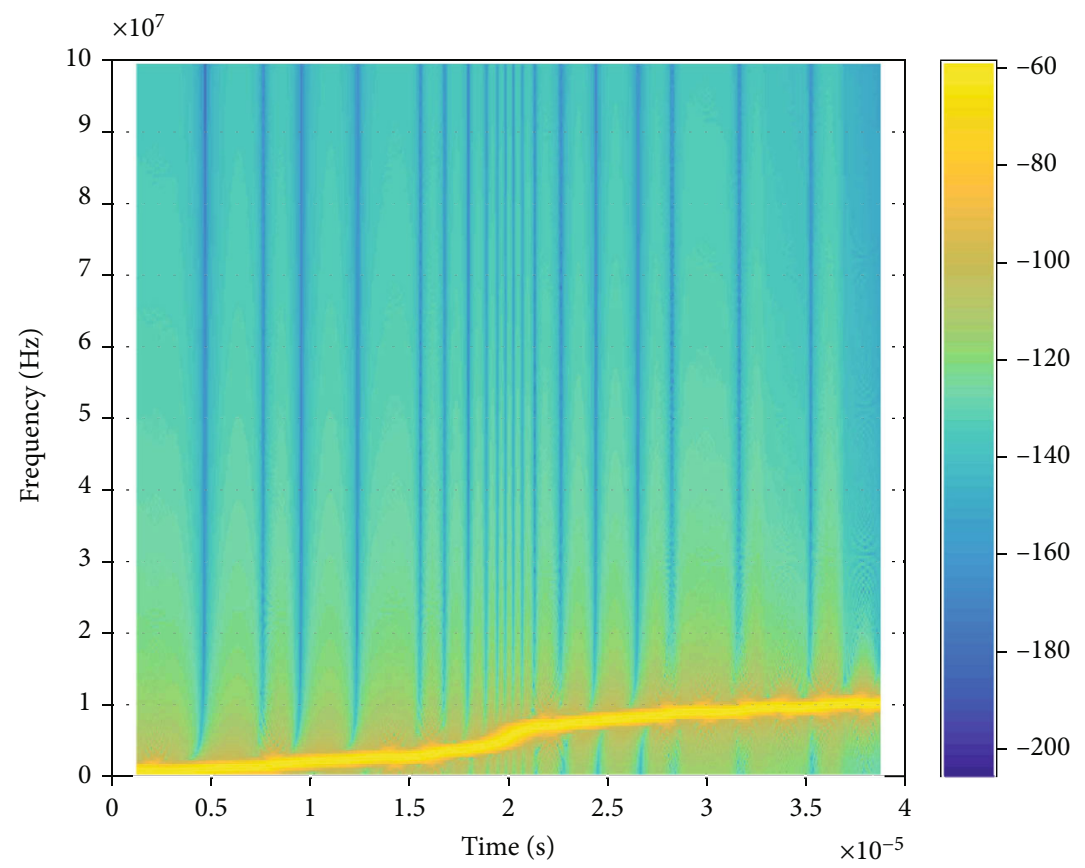

(d)

Figure 15: Comparisons of spectrogram of four signals: (a) NLFM; (b) JRC with 5 bits; (c) JRC with 10 bits; (d) JRC with 20 bits.

As shown in Figure 11, the sidelobes of time AF for LFM signal and LFM-MSK signal [12] are too high, and LFMMSK has poor range resolution for nearby targets. However, JRC signal in this paper takes advantage of the low sidelobe characteristics of time AF for NLFM signal itself, improving the range resolution of the integrated signal, but at the expense of a wider main lobe.

As shown in Figure 12, under the condition $0 \leq N \ll B$. $T_{P}$, with the increase of data carried by JRC signal, the sidelobe of the time AF of the signal gradually also does whereas the range resolution of radar detection gradually deteriorates. It also reflects the compromise between radar and communication, which accords with theoretical analysis.

Figure 13 illustrates that if the communication data increases, the JRC signals and NLFM signal have the same Doppler AF, and hence, they have the same velocity resolution. Our JRC can hold the same velocity resolution without interference of the random data. 
TABLE 3: Communication rate of JRC.

\begin{tabular}{lcccc}
\hline$N$ & $T_{P}$ & $\mathrm{RPI}$ & Rate & Duty \\
\hline 5 & $40 \mu \mathrm{s}$ & $5 \mathrm{kHz}$ & $25 \mathrm{~kb} / \mathrm{s}$ & $20 \%$ \\
10 & $40 \mu \mathrm{s}$ & $5 \mathrm{kHz}$ & $50 \mathrm{~kb} / \mathrm{s}$ & $20 \%$ \\
20 & $40 \mu \mathrm{s}$ & $5 \mathrm{kHz}$ & $100 \mathrm{~kb} / \mathrm{s}$ & $20 \%$ \\
5 & $40 \mu \mathrm{s}$ & $10 \mathrm{kHz}$ & $50 \mathrm{~kb} / \mathrm{s}$ & $40 \%$ \\
10 & $40 \mu \mathrm{s}$ & $10 \mathrm{kHz}$ & $100 \mathrm{~kb} / \mathrm{s}$ & $40 \%$ \\
20 & $40 \mu \mathrm{s}$ & $10 \mathrm{kHz}$ & $200 \mathrm{~kb} / \mathrm{s}$ & $40 \%$ \\
\hline
\end{tabular}

Now, we compare the JRC signal with the original NLFM signal in terms of detection probability. The formula of detection probability was analyzed in Section 3.1. Based on the derivation of equation (29), the detection probability of JRC integrated waveform and the seminal NLFM signal will not change. However, due to the increase of communication data, the spectrum of JRC signal has been expanded, leading to a greater bandwidth. The impact on the JRC signal is a worsening in both the SNR and the detection probability. When the system constant false alarm rate is $10^{-6}$, the detection probability simulation results of JRC with 5 bits and NLFM signals are shown in Figure 14. The results show that both signals have the same detection probability.

4.2. Communication Performance Simulation. Since the MSK modulation communication mode has continuous phase features and the condition $0 \leq N \ll B \cdot T_{P}$ needs to be met in the integrated signal design, the spectrum spread caused by carrying data has almost no impact on the design of the communication receiver. The spectrogram comparison between JRC signals and seminal NLFM signal is shown in Figure 15. Through experimental simulation, the timefrequency diagram of seminal NLFM wave conforms to the curve distribution. When a small amount of communication data are carried, the time-frequency distribution does not change, and the influence of the spectrum diffusion of JRC signal can be ignored. When the amount of data increases, as can be seen from the theoretical analysis in 3.2, the time-frequency diagram has been expanded. Considering that the integrated signal carries a small amount data in this paper, it can be concluded that JRC signal has excellent spectral characteristics. In the case of ideal receiver, the reception of JRC signal is eminent, and our JRC has excellent communication performance.

The communication rate of JRC signal can be calculated from the analysis in Section 3.2. When the carrying amount of unit pulse data meets the conditions, the larger the PRI, the higher the communication rate will be. Therefore, increasing the bandwidth and decreasing the width are considered to increase the PRI while generating a larger time wideband product. Assuming that the bandwidth is $10 \mathrm{MHz}$ and time width is $40 \mu \mathrm{s}$, the duty ratio of the integrated signal is $40 \%$, and the signal pulse repetition rate is $10 \mathrm{kHz} / \mathrm{s}$. In this case, the communication rate of the integrated signal is $N \times 10$ $\mathrm{kHz} / \mathrm{s}$. When $N$ is 20 , the communication rate is $200 \mathrm{kHz} / \mathrm{s}$. The communication rate of JRC signal can be improved by proper design of bandwidth, time width, and carrying data of unit pulse. By adjusting the appropriate parameters, a large communication rate can be obtained. The communication rate of JRC is shown in Table 3.

\section{Conclusions}

In order to solve the problem of high autocorrelation sidelobe and poor nearby target detection performance of LFM-MSK integration signal, this paper proposes an NLFM-MSK integration signal which combines NLFM signal and MSK signal and describes the generation process and time-domain characteristics of JRC signal in detail. Through the signal AF analysis, detection probability derivation, spectrum characteristics, and communication speed characteristic analysis, it is verified that the proposed JRC signal has superior detection performance and good communication performance under the condition $0 \leq N \ll B$. $T_{P}$. Compared with the LFM-MSK signal [11], it has the same detection probability and bit error rate. Meanwhile, it improves the capability of range resolution and nearby multitarget detection in radar detection in the low data volume. It is also proved that the lightning communication rate can be adjusted by changing the signal bandwidth, and our JRC signal is an efficient and flexible radar-communication integrated signal. In the future, we will consider on exploring a variety of NLFM signals used in the field of JRC, whose communication performance and radar performance will be analyzed.

\section{Data Availability}

The data used to support the findings of this study are available from the corresponding author upon request.

\section{Conflicts of Interest}

The authors declare no conflict of interest.

\section{Acknowledgments}

This work was supported by the Fundamental Research Funds for the Central Universities 2021RC260.

\section{References}

[1] B. Hong, W. Wang, and C. Liu, "Ergodic interference alignment for spectrum sharing radar-communication systems," IEEE Transactions on Vehicular Technology, vol. 68, no. 10, pp. 9785-9796, 2019.

[2] B. Paul, A. R. Chiriyath, and D. W. Bliss, "Survey of RF communications and sensing convergence research," IEEE Access, vol. 5, pp. 252-270, 2017.

[3] M. Kiviranta, I. Moilanen, and J. Roivainen, "5G radar: scenarios, numerology and simulations," in 2019 International Conference on Military Communications and Information Systems (ICMCIS), pp. 1-6, Budva, Montenegro, 2019.

[4] M. Arik and O. B. Akan, "Capacity analysis for joint radarcommunication capable coherent MIMO radars," Physical Communication, vol. 40, article 101062, 2020. 
[5] A. Gameiro, D. Castanheira, J. Sanson, and P. P. Monteiro, "Research challenges, trends and applications for future joint radar communications systems," Wireless Personal Communication, vol. 100, no. 1, pp. 81-96, 2018.

[6] H. Ma, J. Wang, X. Sun, T. W. Hou, and W. X. Jin, "Joint radar-communication relying on spread spectrum: a hybrid chaotic sequence design," Wireless Communications and Mobile Computing, vol. 2020, Article ID 8848172, 22 pages, 2020.

[7] W. Zhao, Y. Zhang, and H. Zhang, "A burst SC-FDE scheme for high-speed communication based on radar," in MILCOM 2013-2013 IEEE Military Communications Conference, pp. 1541-1546, San Diego, CA, USA, 2013.

[8] L. Han and $\mathrm{K} . \mathrm{Wu}$, "24-GHz joint radar and radio system capable of time-agile wireless sensing and communication," in 2011 IEEE MTT-S International Microwave Symposium, pp. 1-4, Baltimore, MD, USA, 2011.

[9] X. Liu, T. Huang, N. Shlezinger, Y. Liu, J. Zhou, and Y. C. Eldar, "Joint transmit beamforming for multiuser MIMO communications and MIMO radar," IEEE Transactions on Signal Processing, vol. 68, pp. 3929-3944, 2020.

[10] W. Zhang and Z. S. He, "Comments on "Waveform optimization for transmit beamforming with MIMO radar antenna array", IEEE Transactions on Antennas and Propagation, vol. 66, no. 11, pp. 6463-6463, 2018.

[11] A. Hassanien, M. G. Amin, Y. D. Zhang, and F. Ahmad, "Dualfunction radar-communications: information embedding using sidelobe control and waveform diversity," IEEE Transactions on Signal Processing, vol. 64, no. 8, pp. 2168-2181, 2016.

[12] L. Zhipeng, C. Xingbo, W. Xiaomo, S. Xu, and F. Yuan, "Communication analysis of integrated waveform based on LFM and MSK," in IET international radar conference 2015, pp. 1-5, Hangzhou, 2015.

[13] X. Chen, Z. Liu, Y. Liu, and Z. Wang, "Energy leakage analysis of the radar and communication integrated waveform," IET Signal Processing, vol. 12, no. 3, pp. 375-382, 2018.

[14] Y. Ni, Z. Wang, Q. Huang, and M. Zhang, "High throughput rate-shift integrated system for joint radar-communications," IEEE Access, vol. 7, pp. 78228-78238, 2019.

[15] M. J. Nowak, Z. Zhang, L. LoMonte, M. Wicks, and Z. Wu, "Mixed-modulated linear frequency modulated radar-communications," IET Radar, Sonar \& Navigation, vol. 11, no. 2, pp. 313-320, 2017.

[16] M. Nowak, M. Wicks, Z. Zhang, and Z. Wu, "Co-designed radar-communication using linear frequency modulation waveform," IEEE Aerospace and Electronic Systems Magazine, vol. 31, no. 10, pp. 28-35, 2016.

[17] S. Y. Nusenu, H. Chen, and W. Wang, "OFDM chirp radar for adaptive target detection in low grazing angle," IET Signal Processing, vol. 12, no. 5, pp. 613-619, 2018.

[18] M. Li, W. Wang, and Z. Zheng, "Communication-embedded OFDM chirp waveform for delay-Doppler radar," IET Radar, Sonar \& Navigation, vol. 12, no. 3, pp. 353-360, 2018.

[19] Y. Liu, G. Liao, J. Xu, Z. Yang, and Y. Zhang, "Adaptive OFDM integrated radar and communications waveform design based on information theory," IEEE Communications Letters, vol. 21, no. 10, pp. 2174-2177, 2017.

[20] F. Liu, L. Zhou, C. Masouros, A. Li, W. Luo, and A. Petropulu, "Toward dual-functional radar-communication systems: optimal waveform design," IEEE Transactions on Signal Processing, vol. 66, no. 16, pp. 4264-4279, 2018.
[21] F. Liu, C. Masouros, A. P. Petropulu, H. Griffiths, and L. Hanzo, "Joint radar and communication design: applications, state-of-the-art, and the road ahead," IEEE Transactions on Communications, vol. 68, no. 6, pp. 3834-3862, 2020.

[22] F. Liu, C. Masouros, A. Li, H. Sun, and L. Hanzo, "MU-MIMO communications with MIMO radar: from co-existence to joint transmission," IEEE Transactions on Wireless Communications, vol. 17, no. 4, pp. 2755-2770, 2018.

[23] C. B. Barneto, S. D. Liyanaarachchi, T. Riihonen, L. Anttila, and M. Valkama, "Multibeam design for joint communication and sensing in 5G new radio networks," in ICC 2020-2020 IEEE International Conference on Communications (ICC), pp. 1-6, Dublin, Ireland, 2020.

[24] Y. Zhang, Q. Li, L. Huang, C. Pan, and J. Song, "A modified waveform design for radar-communication integration based on LFM-CPM," in 2017 IEEE 85th vehicular technology conference (VTC spring), pp. 1-5, Sydney, NSW, 2017.

[25] Q. Li, K. Dai, Y. Zhang, and H. Zhang, "Integrated waveform for a joint radar-communication system with high-speed transmission," IEEE Wireless Communications Letters, vol. 8, no. 4, pp. 1208-1211, 2019.

[26] Y. Zhang, W. Wang, R. Wang, Y. Deng, G. Jin, and Y. Long, "A novel NLFM waveform with low sidelobes based on modified Chebyshev window," IEEE Geoscience and Remote Sensing Letters, vol. 17, no. 5, pp. 814-818, 2020.

[27] R. Ghavamirad and M. A. Sebt, "Sidelobe level reduction in ACF of NLFM waveform," IET Radar, Sonar \& Navigation, vol. 13, no. 1, pp. 74-80, 2019.

[28] M. Jiang, G. Liao, Z. Yang, and Z. Wang, "Signal design for integrated radar and communication based on NLFM-CPM," Systems Engineering and Electronics, vol. 41, no. 1, pp. 35-42, 2019.

[29] A. Balleri and A. Farina, "Ambiguity function and accuracy of the hyperbolic chirp: comparison with the linear chirp," IET Radar, Sonar \& Navigation, vol. 11, no. 1, pp. 142-153, 2017.

[30] Y. Liu, N. Cao, M. Mao, and G. Li, "Novel radar and communication integration waveform based on shaped octal phaseshift keying modulation," Physical Communication, vol. 38, no. 2, article 100985, 2020.

[31] Z. Liu, W. Zhang, and S. Xu, "Implementation on the integrated waveform of radar and communication," in 2013 International Conference on Communications, Circuits and Systems (ICCCAS), pp. 200-204, 2013.

[32] U. Majumder, M. R. Bell, and M. Rangaswamy, "Design and analysis of radar waveforms achieving transmit and receive orthogonality," IEEE Transactions on Aerospace and Electronic Systems, vol. 52, no. 3, pp. 1056-1066, 2016.

[33] B. R. Mahafza, "Radar systems analysis and design using MATLAB," in pp. 176-183, Publishing House of Electronics Industry, Beijing, China, 3rd ed edition, 2016.

[34] B. K. Chalise, M. G. Amin, and B. Himed, "Performance tradeoff in a unified passive radar and communications system," IEEE Signal Processing Letters, vol. 24, no. 9, pp. 1275-1279, 2017.

[35] P. Koanantakool, A. Azad, A. Buluç et al., "Communicationavoiding parallel sparse-dense matrix-matrix multiplication," in 2016 IEEE International Parallel and Distributed Processing Symposium (IPDPS), pp. 842-853, Chicago, IL, USA, 2016. 